\title{
Ladri di vestiti in Sicilia: Adrano, Efesto, i Palici e la giustizia divina
}

Thieves of Clothes in Sicily: Adrano, Hephaestus, the Palici and Divine Justice

\section{Gian Franco Chiai}

\section{(2) OpenEdition \\ - Journals}

\section{Edizione digitale}

URL: http://journals.openedition.org/mythos/285

DOI: $10.4000 /$ mythos.285

ISSN: 2037-7746

\section{Editore}

Salvatore Sciascia Editore

\section{Edizione cartacea}

Data di pubblicazione: 1 décembre 2018

Paginazione: 9-46

ISBN: 978-88-8241-501-3

ISSN: $1972-2516$

Notizia bibliografica digitale

Gian Franco Chiai, «Ladri di vestiti in Sicilia: Adrano, Efesto, i Palici e la giustizia divina », Mythos [Online], 12 | 2018, online dal 24 septembre 2019, consultato il 28 septembre 2019. URL : http:// journals.openedition.org/mythos/285; DOI : 10.4000/mythos.285

Mythos 


\section{Ladri di vestiti in Sicilia: Adrano, Efesto, i Palici e la giustizia divina}

\section{Gian Franco Chiai}

\begin{abstract}
Lo scopo di questo studio è quello di proporre in primo luogo una comparazione tra le tradizioni locali dei santuari di Adrano, di Efesto ad Enna e dei Palici e le situazioni di vita quotidiana narrate nelle iscrizioni confessionali e nelle preghiere per la giustizia, ai fini di trovare punti di contatto ed analogie che permettano di ricostruire la presenza di comuni modelli nella scelta e nell'utilizzo di determinate forme di comunicazione religiosa. In secondo luogo, intendo fare delle considerazioni sulla maniera in cui il contatto con i Greci e con la loro cultura abbia fatto in modo che nuove forme di comunicazione religiosa venissero introdotte nell'ambito dei culti locali, grazie alle quali proprio l'elemento locale non viene obliato o cancellato, ma grazie ad un processo di ridefinizione, che trova paralleli interessanti anche in altre regioni del mondo greco e romano, continui ad esistere in una nuova veste.
\end{abstract}

\section{Parole chiave}

Giustizia divina - ellenizzazione - Sicilia • Greci • popoli locali

\section{Keywords}

Divine Justice - Hellenisation • Sicily • Greeks • local people

\begin{abstract}
Riassunto
This study aims at making a comparison between the local traditions of the sanctuaries of Adrano, of Efesto in Enna and the Palici and the situations of daily life narrated in the confessional inscriptions and in the prayers for justice. My purpose is to find points of contact and analogies that allow us to reconstruct the presence of common models in the choice and use of certain forms of religious communication. Secondly, I intend to make some considerations on the way in which the contact with the Greeks and their culture has made sure that new forms and media of religious communication were introduced within the local cults. The new media of religious communication made possible that the local religious elements were not obliterated, but thanks to a process of redefinition, which finds interesting parallels also in other regions of the Greek and Roman world, these continued to exist in a new (Greek) habit.
\end{abstract}

a recente monografia di Nicola Cusumano "Adrano, Efesto e i Palici" ha tra i suoi meriti quello di aver posto in evidenza il ruolo importante che l'idea di giustizia divina aveva nell'ambito di questi culti indigeni nella Sicilia antica ${ }^{1}$. Le tradizioni raccolte ed esaminate nel volume mostrano, infatti, come persone che osavano commettere un crimine, compiere un atto di spergiuro o anche avvicinarsi al luogo di culto di queste divinità venissero punite dal potere divino, subendo una pena forse non commisurata alla gravità del loro reato. Si tratta di una situazione che, a mio avviso, ricorda i racconti delle iscrizioni confessionali della Frigia e della Lidia di epoca imperiale, così come alcuni dei crimini evocati nelle cosiddette "preghiere per la giustizia" (prayers for justice). Cusumano offre inoltre un'analisi del culto ordalico dei Palici attraverso l'uso del modello teorico cosiddetto del "middle ground":

* Ringrazio Nicola Cusumano e Daniela Bonanno per i preziosi suggerimenti datimi nel corso dell'elaborazione di questo lavoro, che dedico a quella nostra amicizia che ci lega da anni. Ringrazio anche il mio amico Marco Palone (Edimburgo) per avermi aiutato a liberare il mio testo da diverse interferenze teutoniche.

1 Cusumano 2015.

2 Il concetto di "middle ground" venne introdotto da Richard White (White 2011) nello studio delle interazioni culturali tra popolazioni indiane e coloni inglesi nella regione dei Grandi Laghi, al confine tra gli Stati Uniti ed 
un'analisi che gli permette di condurre interessanti riflessioni sul modo in cui l'introduzione e l'uso della scrittura avessero contribuito a ridefinire un antico rituale locale.

Lo scopo di questo studio è, come in parte sopra accennato, quello di proporre in primo luogo una comparazione tra le tradizioni locali di questi santuari della Sicilia e le situazioni di vita quotidiana narrate nelle suddette iscrizioni confessionali e nelle preghiere per la giustizia, ai fini di trovare punti di contatto ed analogie che permettano di ricostruire la presenza di comuni modelli nella scelta e nell'utilizzo di determinate forme di comunicazione religiosa. In secondo luogo, intendo fare delle considerazioni sulla maniera in cui il contatto con i Greci e con la loro cultura abbia fatto in modo che nuove forme di comunicazione religiosa venissero introdotte nell'ambito dei culti locali, grazie alle quali proprio l'elemento locale non viene obliato o cancellato, ma grazie ad un processo di ridefinizione, che trova paralleli interessanti anche in altre regioni del mondo greco e romano, continua ad esistere in una nuova veste ${ }^{3}$.

Andando con ordine, in primo luogo prenderò in analisi il contesto del santuario di Adrano e le tradizioni sulla punizione dei ladri di mantelli per mezzo dei cani sacri, soffermandomi anche sul ruolo di questi animali nel santuario di Efesto ad Inessa. Successivamente, verranno considerate le tradizioni sul giuramento di tipo ordalico, che avveniva nel santuario dei Palici; per finire intendo fare delle riflessioni sulla ridefinizione della religione indigena in Sicilia a seguito del contatto con le popolazioni greche. Prima di iniziare, ritengo tuttavia opportuno illustrare brevemente i generi delle iscrizioni confessionali e delle preghiere per la giustizia insieme ai concetti di comunicazione religiosa verticale ed orizzontale.

\section{Le iscrizioni confessionali}

Il termine "iscrizioni confessionali" (Beichtinschriften, confessional inscriptions) si riferisce ad un gruppo di iscrizioni provenienti dai santuari rurali della Frigia e della Lidia di epoca imperiale $e^{4}$, nelle quali al centro della comunicazione religiosa si trova la pubblica confessione di un peccato fatta da un penitente ${ }^{5}$. A causa della sua mancanza, compiuta volontariamente

il Canada, nel periodo compreso tra il 1650 ed il 1815. Il merito dello studioso sta nella fine ricostruzione in cui le culture indigene a seguito del contatto, anche violento, con i coloni inglesi attraverso l'adozione di elementi dall'esterno ridefinirono la propria cultura ed identità, integrando il nuovo nella loro tradizione. Questo concetto è stato messo a frutto da Corinne Bonnet nella ricostruzione dei paesaggi religiosi della Fenicia ellenistica (Bonnet 2014, 29-34, con considerazioni su questo modello). Lo storico Irad Malkin ha ugualmente fatto uso di questo concetto nell'analisi delle interazioni tra Greci ed indigeni nei contesti coloniali in Occidente (MALKIN 1998; ID. 2002; ID. 2004; ID. 2011), così come l'archeologo Luca Cerchiai in relazione ai rapporti tra coloni greci e popolazioni locali nell'antica Pitecussa ed in Campania (Cerchiar 2017). Per una buona sintesi delle moderne teorie coloniali e post-coloniali applicate allo studio della storia antica rimando a GRECO-LOMBARDO 2012 e Sommer 2012.

3 In questo contesto, senza voler approfondire un dibattuto problema di carattere terminologico, mi avvarrò spesso del termine "ellenizzazione". Uso questo termine nella sua accezione generale per designare l'insieme dei processi di contatto e di acculturazione, in cui le popolazioni locali adottano elementi di cultura greca, che possono essere relativi alla sfera della cultura materiale, del sacro, delle istituzioni politiche etc., ridefinendo spesso la propria identità.

4 Va detto che la definizione stessa di iscrizione confessionale è giudicata da alcuni studiosi non soddisfacente, preferendo in alcuni casi quella di iscrizione di riconciliazione, proprio in quanto con l'atto di erezione della stele iscritta il penitente si riconcilia con il proprio dio.

5 La bibliografia sulle iscrizioni confessionali è consistente; in generale rimando a KLAUCK 1996 (che analizza 
o involontariamente ${ }^{6}$, il penitente è stato punito dalla divinità, in genere con una terribile malattia ${ }^{7}$. La pubblica confessione del peccato e l'erezione della stele, sulla quale è stata apposta l'iscrizione con la confessione, rappresentano la via per riconciliarsi con il dio. Una approfondita discussione del carattere di queste iscrizioni non rientra nei fini di questo lavoro. In generale gli studiosi hanno sottolineato l'importanza del modo in cui la religione, attraverso la sapiente costruzione dell'immagine della divinità come giudice onnipotente ed infallibile che siede in cielo $^{8}$, si propone come un' efficace alternativa alla giustizia umana ${ }^{9}$. In altre parole, il timore verso gli dei rappresenta una forma di controllo, che garantisce il rispetto delle norme sociali ed un vivere tranquillo. Altri studiosi si sono, invece, soffermati sulla struttura e sui topoi narrativi presenti in questi testi, che presentano evidenti punti di contatto con gli iamata degli Asklepieia e con le aretalogie di Iside ${ }^{10}$, mostrando l'assunzione di forme di comunicazione religiosa analoghe anche in questi remoti angoli dell'Impero Romano, secondo il modello centroperiferia ${ }^{11}$. Va, infine, ricordato che il rituale della confessione pubblica è attestato anche in altri culti del mondo ellenistico-romano (Iside, Dea Syria etc.) ${ }^{12}$, ma solo in Lidia ed in Frigia risulta essere stato posto per iscritto su pietra.

da un punto di vista linguistico e tematico le corrispondenze con il Nuovo Testamento); CHANIOTIS 1997, ID. 2004, ID. 2009, ID. 2012a (che si è soffermato in particolar modo sulla rappresentazione della giustizia divina e sull'emozionalità che trasuda dai racconti di queste iscrizioni); Petzl 1998; Belayche 2006a-b; EAd. 2007; EAD. 2012 (che ha messo in rilievo i punti di contatto con gli iamata del culto di Asclepio e con le aretalogie di Iside, studiando anche l'idea di regalità divina presente in questi testi). Sui culti rurali della Frigia e della Lidia romana cfr. Petzl 1995; Gnoli-Thornton 1997; de Hoz 1999 (che ha raccolto le iscrizioni di carattere sacro della Lidia); Petzl 2002; HüBner 2003; Dignas 2003; Chiar 2010. Un’edizione dei testi delle iscrizioni confessionali è stata approntata da Georg Petzl (Petzl 1994). Nei successivi venti anni sono stati rinvenuti nuovi testi confessionali: SEG XLVII, 1651, 1654, 1751; LIII, 1158, 1159, 1172-1174, 1182, 1185-1187, 1210, 1211, 1222, 1223; Herrmann - Malay 2007, nr. 46, 47, 51, 52, 54-55, 66, 70, 72, 83-85; Malay 1999, nr. 111, 112, 217; MalaY - Petzl 2017, nr. 77, 81, 82, 83, 116, 119, 120, 121, 123, 131-134, 159, 160, 169, $177,178,187,188$ (l'unica iscrizione confessionale in forma poetica). Su un'iscrizione confessionale rinvenuta a Gerusalemme cfr. SEG LIII, 1852, SEG LVII, 1833.

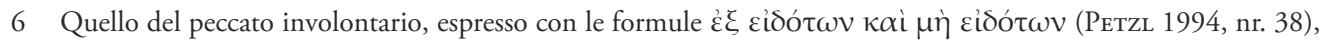

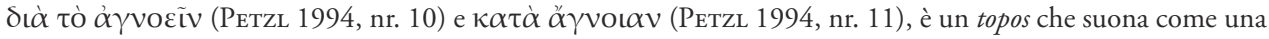
giustificazione di fronte alla divinità e che si incontra in diverse iscrizioni confessionali. Su questo topos, che ricorre anche nella Bibbia e nei testi babilonesi ed ittiti, rimando alle osservazioni di PetTazzoni 1936, 257-277.

7 Le malattie più menzionate sono cecità e disturbi psichici, per un elenco cfr. CHANiotis 1995. Spesso queste malattie, che potevano anche colpire i parenti più stretti del peccatore, potevano portare alla morte. In questo caso l'espiazione del peccato doveva essere condotta dai familiari delle vittime.

8 L'immagine della divinità quale giudice infallibile che siede in cielo ricorre ad esempio in un'iscrizione dalla

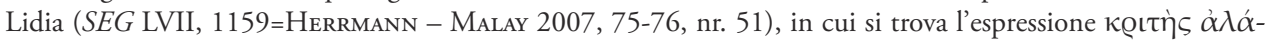

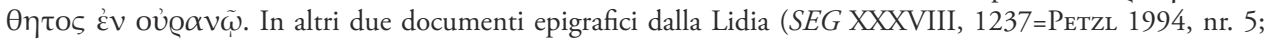
SEG LVII, 1186=HerrmanN - MaLAY 2007, 113-116, nr. 85) si legge di una synatos e di una synkletos theon, ovvero di un'assemblea divina che giudica le mancanze umane, punendole. Su queste testimonianze epigrafiche rimando alle osservazioni di Chaniotis 2012a, 215-223.

9 In questo senso si segnalano in particolare i lavori di Angelos Chaniotis (СHANiotis 1997; ID. 2004); cfr. anche Gordon 2006a.

10 A riguardo rimando a Rostad 2010 (sulle strutture ed i topoi narrativi) e BELAYCHE 2006b; EAD. 2007 (per un confronto con le aretalogie e gli iamata).

11 Sul modello centro-periferia rimando in generale a SCHÖRNER 2005.

12 Cfr. a riguardo con una raccolta ed analisi delle fonti Pettazzoni 1937; come ad esempio Sozomeno (VII, 16, 2-10) testimonia, il rituale della confessione pubblica è attestato anche presso i cristiani, a riguardo cfr. GrAF 2004. 
Le preghiere per la giustizia, la cui definizione si deve a Henk Versnel ${ }^{13}$, sono testi di preghiera, apposti in genere su tavolette di piombo - come supporti sono attestati anche il papiro ${ }^{14}$ e gli ostraka ${ }^{15}$ - che, probabilmente dopo essere stati letti in un contesto rituale in un tempio, venivano deposti nel santuario o affissi sulle sue pareti ${ }^{16}$. Gli autori dei testi, la cui struttura non a caso ricorda quella delle petizioni fatte all'imperatore o ai funzionari in carica ${ }^{17}$, sono persone, per lo più appartenenti agli strati medio-bassi della popolazione, che hanno subito un'ingiustizia e, vista l'inefficacia della giustizia umana, rivolgono la supplica alla loro divinità protettrice, affinché col suo intervento ristabilisca la giustizia, punendo duramente i colpevoli. A differenza delle iscrizioni confessionali, attestate solo in Frigia ed in Lidia, le preghiere per la giustizia sono documentate in molte delle provincie dell'Impero Romano (Egitto, Grecia, Britannia etc.).

Sia le iscrizioni confessionali che le preghiere per la giustizia rappresentano documenti di eccezionale importanza che ci permettono di cogliere importanti aspetti della vita quotidiana nel mondo greco e romano, nonché le speranze che la gente comune riponeva nel potere degli dei.

Veniamo ora al modello di comunicazione religiosa verticale ed orizzontale, quale elaborato in particolare da Ingo Mörth ${ }^{18}$. Ogni testo religioso (sia esso scritto od orale) assolve una funzione comunicativa, ai cui poli stanno un mittente ed un destinatario. La comunicazione religiosa è verticale, dal basso verso l'alto, se il mittente è un mortale ed il destinatario è un dio: inni e preghiere rappresentano testi sacri afferenti a questa categoria. La comunicazione può, tuttavia, avvenire anche dall'alto verso il basso, nel caso che sia la divinità a voler comunicare qualcosa ad un mortale, attraverso ad esempio un'apparizione, un oracolo etc. Per quanto riguarda la comunicazione orizzontale abbiamo a che fare con testi sacri, redatti da mortali, che, in quanto esposti in un luogo accessibile, possono essere letti dagli altri fedeli del dio. A questa categoria appartengono ad esempio le dediche votive, che, proprio in quanto esposte in bella vista in un santuario, attestano da un lato la riconoscenza di un fedele, la cui preghiera è stata esaudita, e dall'altro l'effettiva potenza e presenza della divinità sul posto. Da questo punto di vista le dediche votive possono essere considerate come esempi tanto di comunicazione verti-

13 Versnel 1987a; ID. 1991; ID. 2002; id 2009; ID. 2012. Su questi testi si segnalano i recenti interventi di Mard tin Dreher (Dreher 2010; ID. 2012), il quale sottolinea il carattere emotivo e fortemente soggettivo di queste richieste, proponendo inoltre di sostituire il concetto di "prayers for justice" col termine "Verbrechensflüchen"; da ultimo BerTi 2017, 23-24.

14 Cfr. ad esempio il papiro di Artemisia (PMG LX), il papiro di Neilammon (PMG LI) ed il testo noto come "Fluch des Christen Sabinus" (Bృ̈̈RK 1938=Suppl. Mag. 2, 59).

15 Si tratta di un ostrakon pubblicato per la prima volta da Claudio Gallazzi (GALlazzI 1985=SB XVIII, 13931). Sui diversi supporti scrittori usati per questi testi rimando alle osservazioni di KROpP 2008, 80-82.

16 Le preghiere per la giustizia non sono spesso perfettamente distinguibili da un'altra categoria di testi chiamati "prayers of revenge" (preghiere per la vendetta), che si leggono di solito nelle iscrizioni funerarie di persone assassinate o la cui morte è avvenuta in circostanze sospette. L'esempio più noto è sicuramente quello dell'iscrizione giudaica dell'isola di Reneia (CIJ 70), vicino Delo, in cui il dio giudaico è invocato affinché punisca gli uccisori di una giovane fanciulla. Sulle preghiere per la vendetta rimando agli studi di GRAF 200 I, ID. 2009 (che raccoglie e commenta i testi conosciuti); da ultimo anche Salvo 2012.

17 Questo aspetto fu messo in rilievo per la prima volta da Henk Versnel (Versnel 1991, 71-73); sulle strategie comunicative e sulla struttura testuale delle petitiones cfr. WHITE 1972 (che raccoglie e commenta diversi testi) ed i recenti studi di Stravrianopoulou 2012 e Kotzifou 2012. Un'ampia raccolta di testi delle petizioni ellenistiche di epoca tolemaica in traduzione francese si trova in GUÉrAUd 1931; sulle petizioni all'imperatore cfr. Hauken 1998; sul tema anche le osservazioni di Herrmann 1990.

18 MÖRTH 1993. 
cale che orizzontale. Verticale, in quanto viene comunicato al dio l'adempimento del voto, in seguito alla grazia ricevuta. Orizzontale, dal momento che gli altri visitatori del tempio possono leggere la dedica. In questo contesto possiamo aggiungere che un alto numero di dediche votive, esposte in un santuario, può essere valutato anche in relazione alla concorrenza religiosa tra centri templari ${ }^{19}$. Infatti, dal momento che le divinità delle religioni politeistiche non sono onnipresenti, questi testi attestano che il dio ha caro il luogo del tempio e che una supplica qua rivolta al dio ha più possibilità di venire ascoltata ed esaudita. Da questo punto di vista non è un caso che il numero di dediche votive in pietra (anche la scelta di questo supporto imperituro non è casuale) aumenti in maniera esponenziale soprattutto a partire dall'età ellenistica, quando la concorrenza religiosa diventa più forte, anche in seguito all'arrivo di nuove divinità dall'Oriente ellenistico.

Anche le iscrizioni confessionali assolvono una funzione comunicativa tanto verticale che orizzontale: la divinità stessa pretende l'erezione della stele da un lato quale riconoscimento della sua potenza e dall'altro quale monito agli altri mortali, affinché non commettano lo stesso peccato, sfidando la sua potenza ${ }^{20}$.

\section{Adrano e la sua famiglia}

Del dio Adrano abbiamo non molte notizie, che troviamo sparse in autori greci a partire essenzialmente dal V sec. a.C. ${ }^{21}$. Questa divinità veniva percepita come locale (Eliano userà, come vedremo, l'espressione epichorios daimon), connessa alla montagna ed al fuoco dell'Etna, elemento questo che permetterà una sua identificazione con Efesto nella neo-fondazione di Etna. Il cane è il suo animale sacro ed è anche il tramite con cui il dio manifesta la sua volontà agli umani ${ }^{22}$; tramite questo animale Adrano esplica anche la sua funzione di "giudice divino", che punisce i malfattori ed allontana i non puri di animo dal suo tempio. Questa sua funzione quale "giudice divino" e la sua connessione con l'aristocrazia sicula guerriera ha probabilmente fatto in modo che la statua cultuale del dio nella città di Adrano mutuasse elementi dell'iconografia greca di Ares, quale ad esempio la lancia e forse anche l'elmo, se prendiamo in considerazione la raffigurazione del dio sulle coniazioni dei mercenari Mamertini ${ }^{23}$. In queste

19 Su questo importante fenomeno cfr. Gardan 1992, 1-22; Gladigow 2005, 125-160; Chaniotis 2008; Chiai 2008; Chaniotis 2010 ed i contributi in ENGEL - VAN Nuffelen 2014.

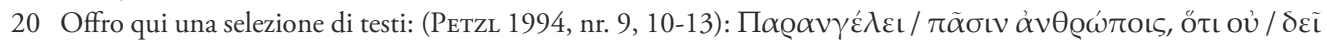

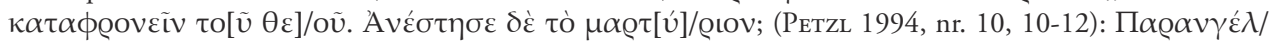

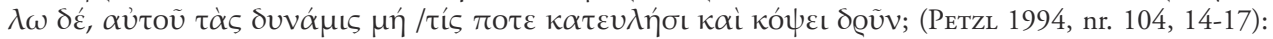

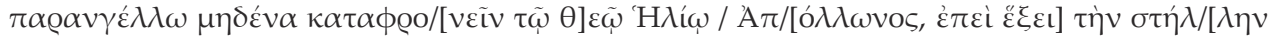

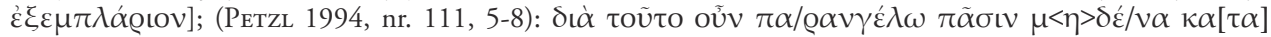

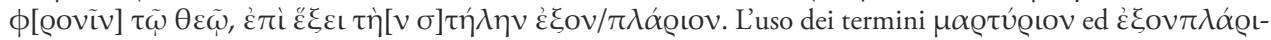
ov (prestito dal latino exemplarium) enfatizza il carattere didattico e morale di questi testi.

21 Su Adrano cfr. Ciaceri 1911, 8-15 (al quale si deve un'importante disamina delle fonti letterarie); tra i lavori più recenti si segnalano soprattutto gli studi di Nicola Cusumano (Cusumano 1990; ID. 1992; ID. 2006; ID. 2015), ai quali si rimanda per la bibliografia precedente e per la storia degli studi. Sull'argomento cfr. anche Morawiecki 1995 e Gagliano 2012.

22 Sul ruolo dei cani nei culti in Sicilia cfr. Ciaceri 1911, 122-133; ed il più recente studio di Cusumano 2004.

23 Su queste emissioni, datate intorno al 288 a.C. (cfr. B.V. Head, Historia nummorum, Oxford 1887, 137), cfr. osservazioni in MoRAWIECKI 1995, 32-39; in generale sugli aspetti iconografici cfr. CANCIANI 1981. 
monete troviamo, infatti, sul dritto la testa barbuta della divinità con un elmo, mentre sul rovescio vediamo un cane, animale, come detto, a lui sacro. Il fatto che dei mercenari scelgano di raffigurare proprio questo dio locale può essere considerato da un lato connesso al suo carattere guerriero e dall'altro, forse, alla loro volontà di interloquire con l'elemento indigeno. Inoltre, anche la sua identificazione con Efesto, fabbricatore di armi per dei ed eroi, collima col suo aspetto guerriero. Tanto l'evidenza archeologica che le fonti letterarie sembrano tacere riguardo ad un pendant femminile del dio. Secondo una tradizione tramandata da Esichio, Adrano sarebbe stato il padre dei Palici, la cui madre sarebbe stata la ninfa delle acque, Talia ${ }^{24}$. Secondo un'altra genealogia, di stampo più greco, i genitori di queste divinità sarebbero stati Zeus e Talia, considerata figlia di Efesto ${ }^{25}$. Un'identificazione che potrebbe far presumere che in una certa data da parte dei Greci avvenne, forse, un tentativo di assimilare Adrano con Zeus. $\mathrm{Va}$, infine, ricordata una terza tradizione, secondo la quale i Palici sarebbero stati generati da Efesto e da Etna, figlia di Oceano ${ }^{26}$. Come Adrano, anche i Palici sono connessi al fuoco ed alla montagna; e come Adrano, anche i Palici sono responsabili per l'ordine sociale e morale. Nel loro santuario, infatti, si recano quanti hanno in corso delle contese di varia natura giuridica; in questo luogo si presta un giuramento ordalico, nell'ambito del quale chi ha dichiarato il falso viene punito con la morte o con una terribile malattia.

Le tradizioni note, nel complesso, seppure trasmesse per tramite greco, permettono di intravvedere la presenza di una sorta di sacra famiglia con a capo una divinità maschile, Adrano, connessa con la guerra e con il fuoco dell'Etna, che si unisce ad una divinità femminile, Talia, legata ad una fonte ed all'acqua, dalla cui unione nascono due gemelli divini. Anche in questo caso incontriamo elementi compatibili con i topoi delle tradizioni mitiche greche e per questo facilmente integrabili in queste.

\section{Adrano e Timoleonte}

Come noto, la città di Adrano venne fondata nel 400 a.C. dal tiranno siracusano Dionisio il Vecchio alle pendici dell'Etna, per consolidare il controllo sul territorio ${ }^{27}$. Il centro rappresentava, infatti, un vitale punto strategico, che garantiva il controllo del Simeto e della città di Centuripe. La fondazione avvenne, probabilmente, nei pressi di un preesistente complesso

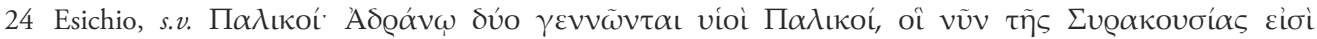

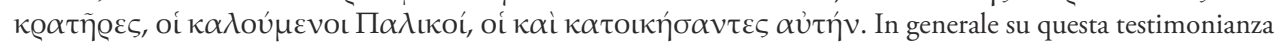
e sulle varie interpretazioni proposte per questa divinità con una bibliografia sull'argomento cfr. CusumANO 2015, 39-42; un'approfondita discussione sulle fonti in Cusumano 1990; ID. 1992, 164-172, 181-185 (che con prudenza propone di vedere in Filisto la fonte del lemma esichiano).

25 Questa tradizione viene tramandata da Stefano di Bisanzio (s.v. Palike).

26 Si tratta di un frammento dello storico Sileno (FGrHist 175, F 3), citato da Stefano di Bisanzio. Secondo Servio (ad Aen. IX, 581), Giove si sarebbe unito alla ninfa Etna e dalla loro unione sarebbero nati i Palici.

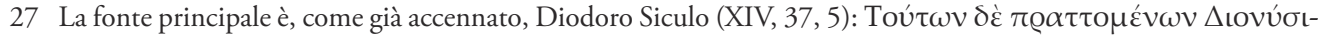

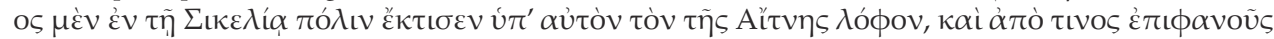

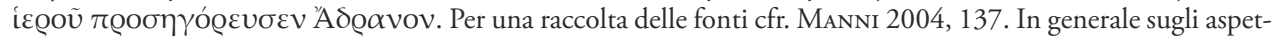
ti archeologici rimando ai documentati studi di Gioconda Lamagna (LAMAGNA 2009a; EAD. 2011; ed i diversi contributi in LAMAGNA 2009b); sulle iscrizioni anelleniche rinvenute nel territorio della città cfr. MANGANARO 1961 (che attribuisce al santuario di Adrano un carattere federale, similmente a quello dei Palici) e la recente messa a punto di Agostiniani 2009. 
templare dedicato al dio, come il nome del fiume Adranon lascerebbe ipotizzare ${ }^{28}$. Secondo una tradizione locale tramandata da Plutarco (Tim. XII, 2-3; XVI, 5-6), nel 344 a.C. Timoleonte di Corinto ${ }^{29}$, marciando con le sue truppe alla volta di Siracusa, dopo aver sbaragliato nel territorio di Adrano l'esercito di Iceta, tiranno di Leontini, sarebbe entrato ad Adrano. Durante lo scontro le porte del santuario di Adrano si sarebbero aperte da sole, mentre il volto della statua del dio avrebbe sudato e la sua lancia si sarebbe mossa: questi prodigiosi avvenimenti vennero interpretati come un messaggio del dio, che in questo modo avrebbe manifestato la sua volontà di aprire le porte della città a Timoleonte. Certamente non è casuale che, proprio a partire dall'epoca timoleontea, questo centro assume da un punto di vista archeologico una propria fisionomia, mentre del periodo precedente, denominato dionisiano, restino solo poche tracce $^{30}$. Procediamo ora ad analizzare i paragrafi 2 e 6 del testo di Plutarco.

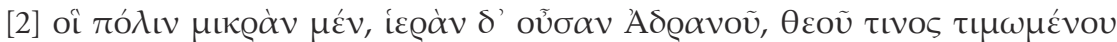

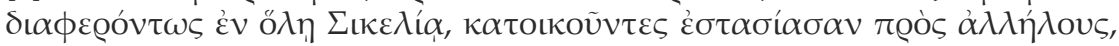

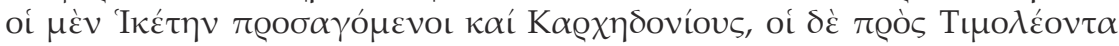

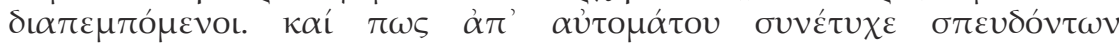

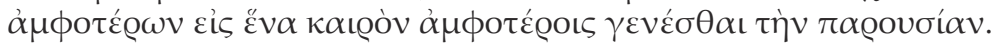

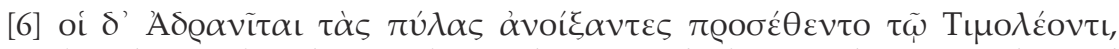

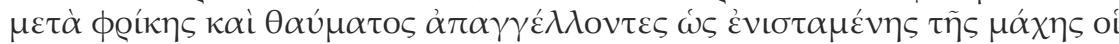

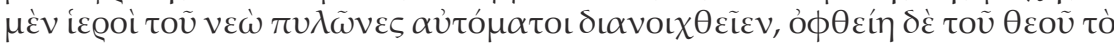

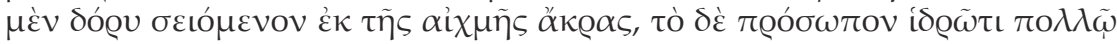
@é́ $\mu \varepsilon v o v$.

[2] (Gli Adraniti), che abitano una piccola città, che è sacra ad Adrano, un dio particolarmente onorato in tutta quanta la Sicilia, erano in discordia tra loro. Alcuni, infatti, parteggiavano per Iceta ed i Cartaginesi, mentre altri erano per Timoleonte. Come per coincidenza avvenne che entrambi i contendenti, affrettandosi, finissero per giungere nello stesso momento in quel luogo.

[6] (Dopo la sconfitta di Iceta) gli Adraniti, aprendo le porte, andarono incontro a Timoleonte, narrando con timore e meraviglia che, mentre aveva luogo la battaglia, le sacre porte del tempio di fossero aperte da sole e che fosse stata vista la lancia del dio scuotersi dall'estremità della punta, mentre il suo viso grondava di molto sudore.

Plutarco definisce Adrano come una piccola città, la cui importanza era dovuta al fatto di essere sacra ad una divinità, particolarmente adorata presso le popolazioni indigene della Sicilia. La particella $\delta \dot{\varepsilon}$ che precede il participio oũ $\sigma \alpha v$ sembra enfatizzare lo stato particolare

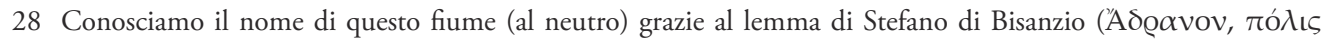

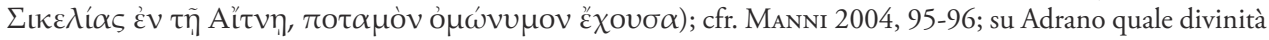
fluviale, con argomenti non sempre convincenti cfr. MoRAWIECKI 1995, 39-42 con bibliografia precedente.

29 Sugli avvenimenti legati all'arrivo di Timoleonte in Sicilia rimando, in generale, alla chiara ricostruzione storica di Sordi 1980 e di De VIdo 2013, 69-79. Tra gli studi più recenti sulla figura di questo condottiero cfr. De Vido 2011.

30 La situazione viene così riassunta da LAmagna 2011, 57: «Infatti, le fasi di vita anteriori a questo periodo, quelle, per intenderci, relative ai periodi successivi alla fondazione dionigiana, sono purtroppo ancora oggi estremamente evanescenti, essendo legate a pochi frustuli di strutture murarie rinvenute una trentina di anni fa nell'area dell'abitato e ad alcuni settori della cinta muraria.». 


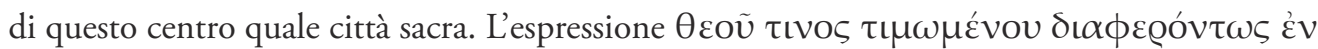
ő $\lambda \eta \eta \Sigma \kappa \varepsilon \lambda$ í $_{1}$ si riferisce, verosimilmente, a tutta quanta quella parte delle Sicilia non greca,

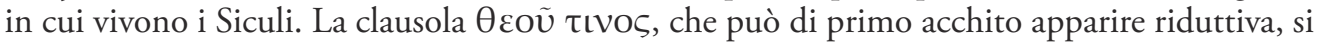
capisce meglio se si riflette sul fatto che i lettori di Plutarco, probabilmente, non conoscevano questa antica divinità sicula. Gli Adraniti, anche con una certa spregiudicatezza politica, dopo aver appreso della sconfitta di Iceta, in maniera plateale aprono le porte della città e vanno

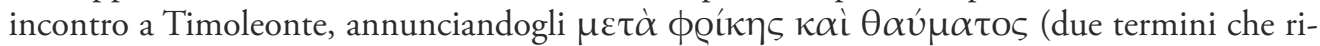
corrono spesso a descrivere le reazioni dei mortali di fronte alle apparizioni divine ${ }^{31}$ ) il prodigio avvenuto. Plutarco tramanda, inoltre, un particolare iconografico molto interessante: la statua cultuale del dio sarebbe stata una statua armata di lancia. Si tratta di un elemento iconografico che sembrerebbe rimandare al carattere guerresco di questa divinità, ben consono al carattere militare dell'aristocrazia locale ${ }^{32}$; probabilmente quello della lancia, come sopra accennato, è un elemento mutuato dall'iconografia di $\mathrm{Ares}^{33}$. Va detto che questo carattere guerresco ben si adatta anche a quelle forme di intervento divino atte a ristabilire la giustizia, che le tradizioni locali, come vedremo, attribuiscono al dio.

In secondo luogo, di rilievo è anche la collocazione intra-urbana del santuario, intorno al quale, come si evince dalle fonti letterarie, Dionigi fece costruire la città. Si tratta di una collocazione che contrasta con quella degli altri Adraneia conosciuti, quali quelli del Mendolito, di Halaesa $^{34}$, situati in posizione sub-urbana. Questo può essere spiegato col fatto che il santuario dava il nome alla città, di cui il dio era il protettore e, senza dubbio, la divinità più importante.

La tradizione plutarchea presenta, come visto, elementi topici, quali quello della statua che suda e si muove e delle porte del tempio che si aprono e chiudono da sole ${ }^{35}$. Segno che questa tradizione locale venne creata usando i topoi classici dei racconti delle apparizioni divine; e segno anche che la classe sacerdotale locale doveva probabilmente conoscere bene questi racconti, per recepirne i topo $i^{36}$ e creare tradizioni locali proprie. Questo potrebbe fornire un utile indizio anche del fatto che la cultura greca aveva permeato per lo meno gli strati alti della società adranea.

Restando sempre in epoca timoleontea, possiamo brevemente ricordare un episodio della vita del celebre condottiero corinzio, il quale, in procinto di sacrificare ad Adrano all'interno

31 Cfr. Versnel 1987 b.

32 Albanese Procelli 1999, 339; Albanese Procelli 2009, che analizza i bronzi del ripostiglio di Adrano, databili tra il IX-VII sec. a.C., nei quali si rinvengono figure stilizzate di oranti ed i frammenti di una statuetta raffigurante un guerriero. I resti di cinturoni in bronzo decorati, studiati da VASSALLO 1999, 90-109, sembrano attestare la presenza di una ricca aristocrazia guerriera locale.

33 Interpretazione, ad esempio, data da PACE 1945, 520, che propendeva per una identificazione col dio della guerra. Più che di un'identificazione si tratterebbe tuttavia dell'adozione di un elemento iconografico di un'altra divinità, ai fini della creazione di una statua cultuale, i cui attributi iconografici erano consoni ai valori guerreschi dell'aristocrazia locale; in questo senso cfr. le osservazioni di GaGLIANo 2012, 308.

34 Cfr. IG XIV 352 I (latus sinistrum) 54, 62-63, con la menzione di un'area sacra extraurbana o suburbana chia-

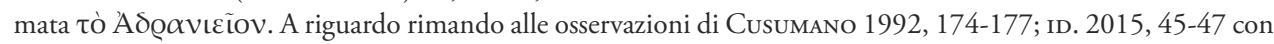
ulteriori riferimenti bibliografici.

35 Cfr. a riguardo osservazioni in MorawIECKI 1995, 34-35, che raccoglie esempi analoghi.

36 In generale sulle apparizioni divine rimando a STRAMAgLia 1999, 7-117 (con una selezione di testi letterari relativi alle apparizioni paranormali); Dickie 2002; Graf 2004b; sul ruolo delle epiphaniai in guerra Chaniotis 2005, 157-160; Platt 2011 (sulle rappresentazioni nell'arte antica); Petridou 2015 (che analizza i testi letterari). Tra gli studi più specifici segnalo Cнаniotis 1998, che studia il famoso "miracolo di Panamara". 
del tempio nell'omonima città, venne incredibilmente salvato da un attentato per opera di due sicari ingaggiati da Iceta, grazie all'intervento provvidenziale di Adrano. Plutarco (Tim. XVI, 5-6) racconta, infatti, che una persona riconobbe nell'attentatore l'uomo responsabile della morte del padre, provocandone il provvidenziale intervento. In questo caso, nel vero senso della parola, la provvidenza divina sembra proteggere Timoleonte, al quale comunque le tradizioni locali di Adrano attribuiscono un rapporto particolare con il dio protettore del centro.

Anche questa tradizione, di chiara matrice adranea, sembra essere stata creata appositamente da un lato per sottolineare il benvolere di Adrano nei confronti del condottiero corinzio, dall'altro per mostrare il senso di giustizia del dio, che non poteva tollerare un assassinio nel suo santuario e nella sua città: un tale atto sanguinoso avrebbe macchiato, infatti, questo luogo sacro.

\section{Adrano come "Schutzgottheit"}

In questo contesto vorrei soffermarmi anche sul carattere di Adrano quale divinità protettrice della città, che trae il proprio nome da quello del dio. Le divinità protettrici delle città rappresentano ancora oggi una categoria tra le meno studiate. Prescindendo dal libro di Barbara Brackertz pubblicato nel $1976^{37}$, alla quale si deve un'utile raccolta delle fonti letterarie ed epigrafiche, ed alle osservazioni condotte da Angelo Brelich in relazione all'anonima divinità protettrice di Roma ${ }^{38}$, manca al giorno d'oggi un lavoro monografico che analizzi in maniera sistematica questa categoria di divinità. La divinità protettrice di una città, proprio in quanto la più importante di un centro cittadino, mostra spesso di possedere ambiti di competenza che travalicano quelli classici e canonici degli dei di Esiodo. Queste divinità, sotto la cui protezione si trovano i cittadini, la città ed il suo territorio, assumono spesso i tratti di divinità onnipotenti, che possono venir invocate in ogni occasione ${ }^{39}$. Da questo punto di vista la caratteristica di Adrano, quale dio giudice che punisce i malfattori, non dovrebbe stupire. Essa anzi si lascia ben confrontare con la rappresentazione della divinità quale un monarca, che regna sul villaggio e sui suoi abitanti, quale presente in diverse formule di acclamazione, che si leggono all'inizio delle iscrizioni confessionali ${ }^{40}$. Nella suddetta monografia Barbara Brackertz

37 BRACKertz 1976.

38 BRELICH 1949; le tesi dello studioso sono state riprese e sviluppate in anni recenti da Giorgio Ferri (FERRI 2010).

39 In questo contesto riprendo alcune mie osservazioni fatte in relazione al concetto di "Ortsgebundenheit des Göttlichen” (CHIAI 2009, in relazione ai santuari rurali della Frigia di epoca romana, poi ripreso in CHIAI 2013, a proposito dell'interpretazione che Macrobio fa degli epiteti divini derivati da toponimi), per cui il legame speciale che lega una divinità ad un determinato luogo (sia questo un centro urbano, un villaggio o un territorio) fa in modo che in quell'ambito il potere divino travalichi spesso quello delle canoniche competenze degli dei olimpici di Esiodo. Considerazioni analoghe in relazione al culto della Dea Siria anche presso FeLdTKELLER 1994, 64: "Wenn eine Gottheit Schutzgottheit der Polis ist, hat das zur Folge, dass ihre funktionale Geltung in der Polis wesentlich umfangreicher ist als die überregionale Geltung der gleichen Gottheit innerhalb eines polytheistischen Religionssystems.». Osservazioni anche in KaIser 2006, 35-39.

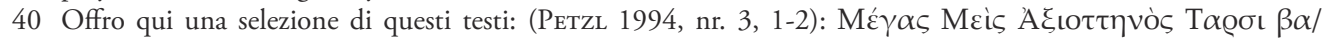

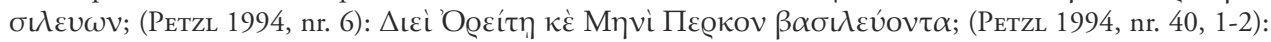

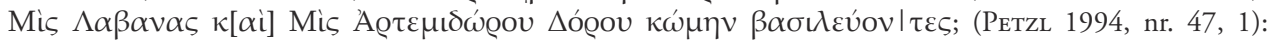

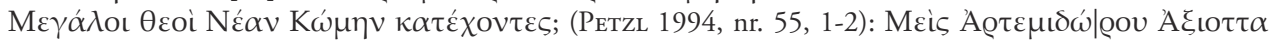

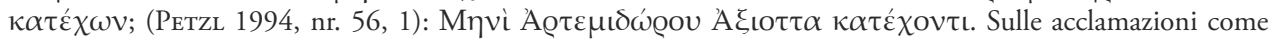
forma di comunicazione religiosa (verticale dal basso verso l'alto) cfr. CHAniotis 2009. Sul concetto di basileia divina in relazione alle divinità dei santuari rurali della Frigia e della Lidia cfr. Belayche 2006b e Chiar 2009. 
identifica cinque caratteristiche principali che una divinità protettiva deve possedere per essere considerata tale ${ }^{41}: 1$ ) al culto della divinità partecipano tutti i cittadini, costituendo questo un elemento centrale dell'identità culturale del centro cittadino; 2) questo culto garantisce prosperità e sicurezza a tutta quanta la comunità; 3) la città ed il suo territorio vengono considerati come possesso della divinità ${ }^{42}$; 4) i successi politici, militari ed economici di una città vengono attribuiti alla divinità protettrice, 5) la quale è la rappresentante della comunità cittadina.

La studiosa elenca poi tutta una serie di altri criteri, che differenziano le "Schutzgottheiten" dagli altri dei adorati in una città ${ }^{43}$, come ad esempio la loro centralità nelle tradizioni mitiche locali, la frequenza nell'onomastica cittadina di nomi teofori da essa derivati, la presenza della sua effigie nelle emissioni locali, e non da ultimo la collocazione del suo santuario nella rocca $^{44}$ o nel centro urbano ${ }^{45}$, anche se abbiamo molti casi di santuari extraurbani ${ }^{46}$. Va poi ricordato che, soprattutto a partire dall'età ellenistica, a queste divinità vengono riferiti epite-

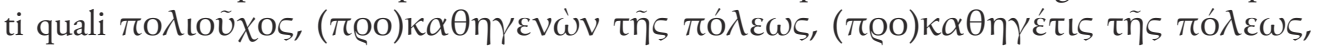

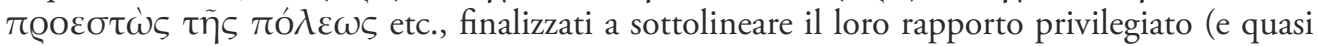
esclusivo) con la città ${ }^{47}$.

Le poche testimonianze di cui disponiamo ci lasciano intravvedere la centralità del culto di Adrano nella città a lui omonima così come la sua morfologia di "Schutzgottheit" della comunità cittadina. Proprio questa caratteristica aiuta meglio a comprendere la sua connessione con la giustizia. In questa nuova definizione della morfologia di Adrano, che nulla toglie alla sua origine autoctona, si può forse scorgere un influsso della religione della polis greca.

\section{I cani sacri di Adrano ed i ladri di mantelli}

Prendiamo ora in analisi il testo della tradizione sui cani sacri di Adrano, quale tramandato presso Eliano (n.a. XI, 20) ${ }^{48}$ :

41 BRACKERTZ 1976, 155.

42 Significativo è in questo senso l'uso del participio $\kappa \alpha \tau \varepsilon \dot{\varepsilon} \chi \omega \mathrm{v}$ in un'iscrizione di Delo in relazione ad Apollo e ad Artemide, le divinità protettrici dell'isola, che secondo una tradizione locale sarebbero nate a Delo: (Syll ${ }^{3}$ 662,

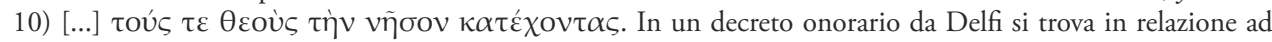

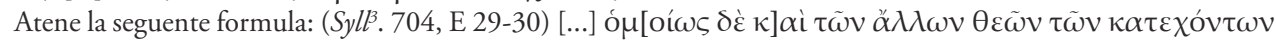

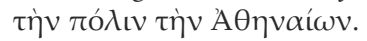

43 Brackertz 1976, 156-160.

44 Nella rocca cittadina era ubicato il santuario della divinità protettrice ad Atene, Tegea, Tebe, Corinto, Trezene, Megara, Lindo e Pergamo.

45 Come nel caso di Priene, Magnesia sul Meandro, Mileto (Delphinion), Afrodisia, Megalopoli e Sicione.

46 Argo, Samo, Epidauro, Kos, Mantinea, Eretria, Elis, Mileto (Didymaion), Colofone, Efeso, Perge e Tegea.

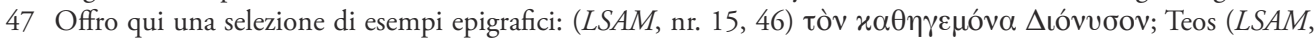

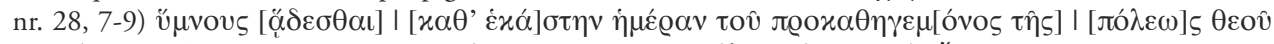

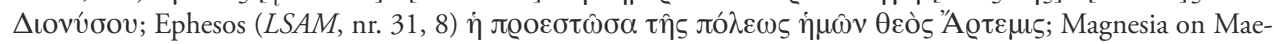

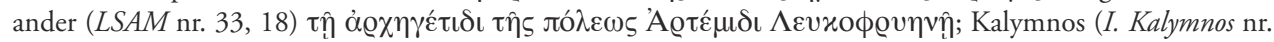

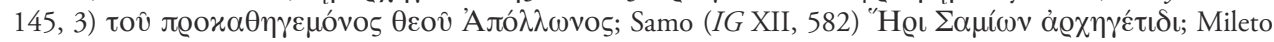

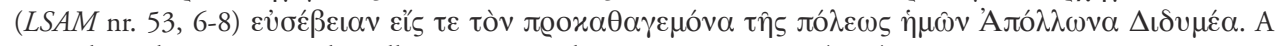
riguardo mi limito a rimandare alle osservazioni di Сналiотіs 2007, 147-149.

48 Tra gli studi più recenti su questa tradizione cfr. Gagliano 2012, 301-306; Cusumano 2015, 54-63, ai quali si rimanda per la bibliografia precedente. 


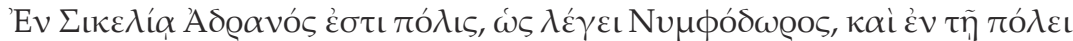

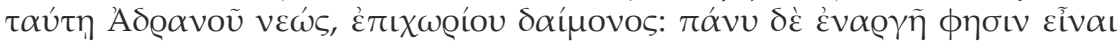

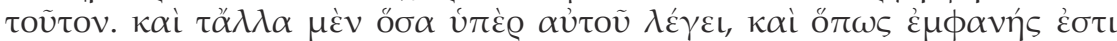

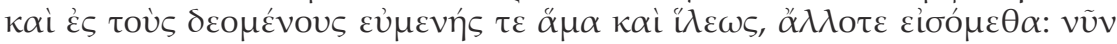

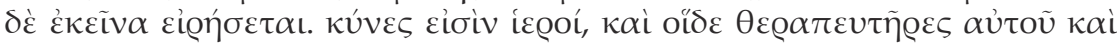

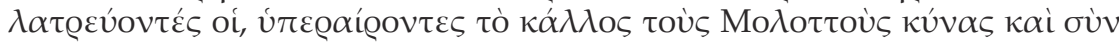

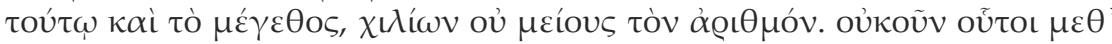

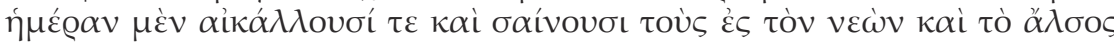

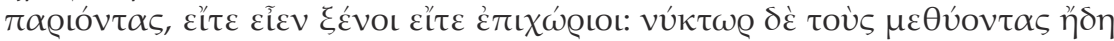

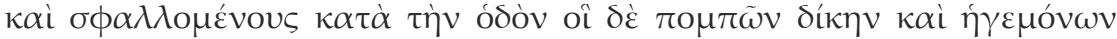

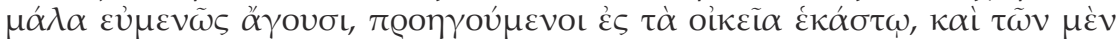

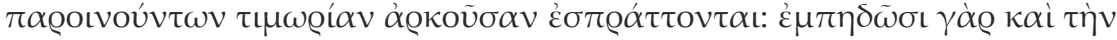

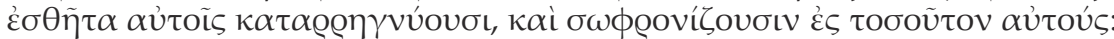

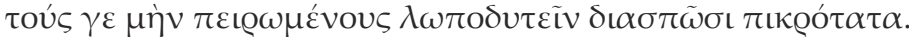

In Sicilia esiste la città di Adrano, come racconta Ninfodoro, ed in questa città si trova il santuario di Adrano, una divinità locale. Si dice che la presenza del dio sia molto tangibile. Quanto altro (Ninfodoro) ci racconta su di lui, in qual modo si renda manifesto e sia benigno e insieme propizio verso coloro che gli si rivolgono nel bisogno, lo conosceremo in un'altra occasione, mentre ora saranno narrati i seguenti fenomeni. Si trovano lì dei cani sacri, suoi servitori e ministri, che superano in bellezza e grandezza i cani molossi, e sono di numero non inferiore al migliaio. Ebbene, questi animali durante il giorno accolgono festosamente dimenando la coda i visitatori che si recano al santuario o al circostante boschetto sacro, e questo senza fare alcuna distinzione tra stranieri e persone del luogo. Diverso è invece il loro comportamento durante la notte, quando essi accompagnano con grande benevolenza, a guisa di scorta, quelli già ubriachi e coloro che non si reggono in piedi lungo il cammino, riconducendoli ciascuno alla propria casa. Fanno però espiare il giusto castigo a coloro che nell'ubriachezza commettono empietà: difatti li assalgono e lacerano la loro veste, e a tal punto li fanno rinsavire. Ma sbranano in maniera crudelissima coloro che provano a $\lambda \omega \pi \operatorname{co\delta } v \varepsilon \tilde{\varepsilon} v$. (Traduzione di Nicola Cusumano)

L'autore narra che questo dio, definito epichorios daimon, rappresenta la divinità più importante nella città che da lui prende il nome, la quale, in virtù di questo fatto, doveva essere considerata come una sorta di hiera polis ${ }^{49}$. Le sue azioni, che - come vedremo - si esplicano in parte tramite l'intervento di questi cani, fanno in modo che la sua presenza fosse avvertita come tangibile. Nel testo di Eliodoro troviamo il termine enarge. Quello dell'enargeia è un concetto molto importante nella retorica antica: esso indica la capacità di rendere vive, visibile e tangibili le fattezze di un oggetto descritto in un'ekphrasis ${ }^{50}$. Tramite il suo utilizzo Eliodoro

49 Un parallelo adducibile è quello della frigia Hierapolis, che in virtù del culto di Apollo e della presenza del Ploutonion, con le relative tradizioni mitiche legate a questo luogo, aveva assunto questo nome, atto a sottolineare la santità del luogo, nel quale la città fu fondata (cfr. D’Andria 2012; Kerschbaum 2014). Un altro esempio anatolico è quello di Aizanoi, che come le ricerche archeologiche hanno mostrato, fu fondata nel luogo in cui sorgeva il santuario di una divinità anatolica, identificata con Zeus. Nel caso di Aizanoi il materiale numismatico mostra come nell'ambito delle tradizioni locali si affermasse che la nascita e la prima infanzia di Zeus avessero avuto luogo nel territorio cittadino (cfr. Jes 2007); questo accadeva in concorrenza con la vicina Laodicea sul Lico e con Tralleis (CHiar 2012, 59-64).

$50 \mathrm{Su}$ questo tema esiste una bibliografia immensa, in generale rimando all'esauriente volume Marino-STAVRU 2015, contenente diversi contributi che studiano questi problemi terminologici. 
vuole probabilmente sottolineare il modo in cui la presenza del dio fosse avvertita come reale e tangibile dagli abitanti del luogo, proprio in virtù delle sue azioni. In questo santuario vivono circa un migliaio di cani, che per bellezza e grandezza superano i cani molossi. Questi animali vengono considerati come i servitori ed i ministri di Adrano. I termini qui utilizzati, therapeuontes e latreuontes, meritano una particolare attenzione. Si tratta di due termini tipici del vocabolario sacro greco, soprattutto a partire dall'età ellenistica ${ }^{51}$, che si riferiscono nel caso di therapeuontes a una persona di fiducia che serve il padrone di casa. In senso traslato questo termine venne applicato a quanti servono la divinità, vista come kyrios/dominus. Il verbo latreuo appartiene invece alla sfera del sacro e designa un servizio reso da una persona nei confronti della divinità. Entrambi i termini, come accennato, sono tipici del vocabolario religioso greco di età ellenistica e potrebbero essere un indizio per una rielaborazione (scritta) delle tradizioni di Adrano in questo periodo. La therapeia e la latreia dei cani si esplica in un servizio di accompagnamento e protezione dei fedeli che visitano il santuario e di difesa di questo nei confronti degli impuri. Durante il giorno, essi accolgono benevolmente i visitatori, mentre nella notte accompagnano a casa quanti ubriachi non possono reggersi in piedi e non sono in grado di ritrovare il camino. Essi mordono quanti, perché ubriachi, tengono un comportamento empio, offendendo il dio e non rispettando la santità del luogo. Questi cani si comportano ancor peggio con coloro che abbiano provato a rubare mantelli, arrivando addirittura a sbranarli in maniera crudele. Il verbo qui usato è $\lambda \omega \tau \operatorname{co\delta } v \tau \varepsilon \tilde{\imath} v$, che propriamente significa ,indossare indumenti d'altri“"52. Anche in questo caso Eliano (o la sua fonte, Ninfodoro) utilizza un termine tecnico molto particolare. A Nicola Cusumano va il merito di aver raccolto tutta una serie di testimonianze letterarie, per la maggior parte di autori attivi nell'Atene del V e del IV sec. a.C.,

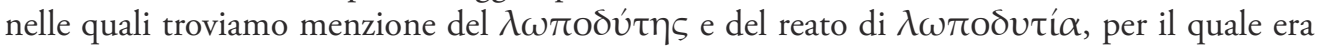
addirittura prevista la pena di morte tramite bastonamento pubblico (apotympanismos) ${ }^{53}$. Dalle testimonianze letterarie si evince la pessima fama che aveva un individuo che si macchiava di un tale reato: un reietto, che non meritava di vivere in mezzo alla società e veniva considerato alla pari dei ladri sacrilegi che spogliavano i templi, dei venditori di schiavi, degli adulteri e degli assassini ${ }^{54}$. Significativo è a riguardo un passo del retore Antifonte, nel quale si menziona

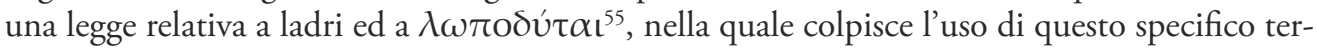

51 Rimando a riguardo alle osservazioni di Pleket 1981, 159-161, 163-166.

52 Il significato di questa forma verbale composta si comprende meglio prendendo in considerazione la seguente

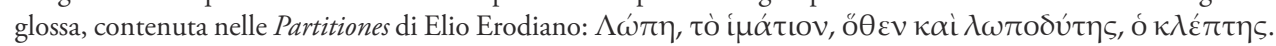

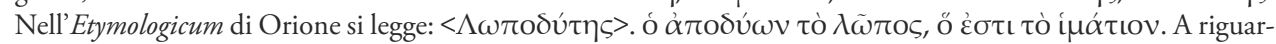
do rimando all'esaustiva trattazione di Cusumano 2015, 55-58.

53 Lys. 10, 14; Lys. 13, 68 (in riferimento alle malefatte della famiglia di Agorato, dei cui tre fratelli uno venne condannato a morte per apotympanismos in quanto reo di aver fatto segnali al nemico in Sicilia, un altro morì in carcere, in quanto condannato per aver svolto commercio illecito di persone libere, un terzo in quanto $\lambda \omega \pi \mathrm{O}-$ $\delta u ́ \tau \eta \varsigma$, dovette subire la pena dell'apotympanismos). Su questo supplizio ci illumina il seguente passo di Fozio:

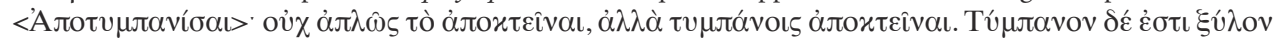
$<\omega ̋ \sigma \pi \varepsilon @>\sigma x u ́ \tau \alpha \lambda o v$. Su questa pena capitale rimando a CANTARELla 1991, 41-53.

54 Xen., Mem. 1,2,62, 4: «Secondo le leggi, infatti, per chi è sorpreso in flagrante a rubare, a rubare il mantello, a tagliare borse, a commettere effrazione, a rendere schiave persone libere, o a rubare nei templi, per tutti costoro è prevista la pena di morte.» (Trad. Santoni 2004); Plat., Resp. 575b: «Per esempio rubano, sfondano i muri, tagliano le borse, rubano i mantelli, spogliano i templi, vendono come schiavi altri cittadini.» (Trad. Lozza 1990). Athen. 6, 12.

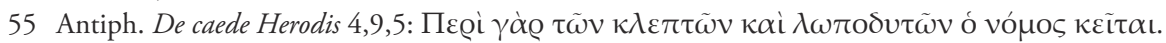


mine per distinguere questi personaggi dai ladri comuni. La cattiva fama di questi personaggi era tale che Platone nelle "Leggi" ( 874 b-d), trattando del diritto alla difesa, affermava che

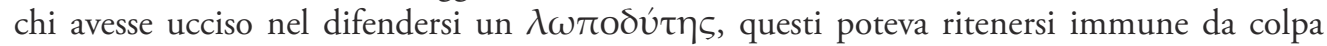

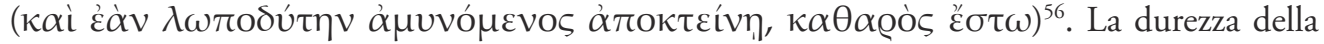
pena prevista può a prima vista apparire spropositata rispetto al reato perpetrato. Va tuttavia posto in rilievo che i mantelli così come in generale gli indumenti che si portano addosso abitualmente e che appartengono alla sfera personale possono possedere per una persona un valore affettivo e simbolico. Proprio in virtù di questo valore possiamo meglio comprendere lo sgomento e la rabbia di una persona che veniva derubata di un proprio indumento personale. A ciò va anche aggiunto il fatto che nell'antichità una persona del ceto medio non doveva possedere un alto numero di indumenti.

La tradizione che leggiamo nel testo di Eliano attribuisce al dio per il tramite dei suoi cani una funzione protettiva e di giudice, che condanna e fa punire in maniera terribile quanti si siano macchiati nel suo santuario di questo indegno reato. Prescindendo dall'assunzione di un codice comportamentale greco in ambito indigeno ${ }^{57}$, quale questa tradizione ci lascia intravvedere, vorrei ora concentrarmi sul significato della punizione dei ladri di mantello tramite l'intervento divino e proporre un parallelo con i testi delle iscrizioni confessionali e delle cosiddette preghiere per la giustizia. Tra i numerosi e coloriti racconti delle iscrizioni confessionali, uno in particolare si presta ad essere considerato in questo contesto.

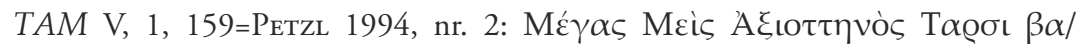

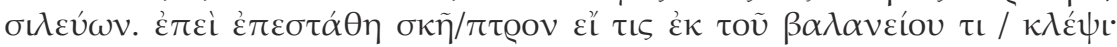

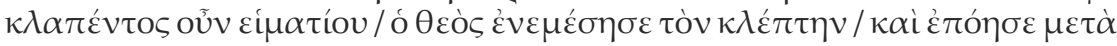

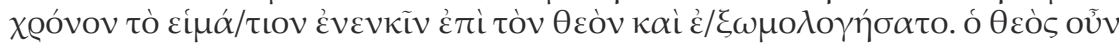

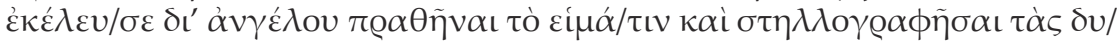

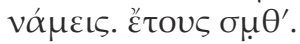

Grande è Men di Axiotta, che regna su Tarsi. Dal momento che uno scettro fu innalzato, nel caso che qualcuno avesse rubato qualcosa dai bagni pubblici, e poiché venne rubato un mantello, il dio punì il ladro e fece in modo che dopo un po' di tempo questi riportasse il mantello nel tempio del dio e confessasse (il suo misfatto). Il dio allora ordinò tramite un angelo di vendere il mantello e col ricavato di far incidere su pietra la manifestazione della sua potenza. Nell'anno 249.

L'iscrizione racconta l'intervento del dio a seguito dell'infrazione del divieto di rubare capi di vestiario da un bagno pubblico. In questo caso il ladro di mantelli venne punito molto probabilmente, come la maggior parte delle iscrizioni confessionali attestano, con una malattia, che lo costrinse a riportare la refurtiva nel tempio del dio e a rendere qui una pubblica confessione del suo misfatto. Il rito dell'innalzamento dello scettro, del quale troviamo menzione in diverse iscrizioni sacre delle Lidia e della Frigia di epoca romana, si riferisce ad un rituale locale, di cui purtroppo i testi epigrafici non forniscono tanti particolari ${ }^{58}$. Probabilmente, lo scettro

56 Per una discussione delle altre testimonianze rimando al già citato studio di Cusumano 2015, 55-63.

57 Rimando sempre alle osservazioni di Cusumano 2015, 63-66.

58 Raccolgo qui alcuni esempi epigrafici tratti dalle formule di maledizione delle iscrizioni funerarie dalla Lidia e

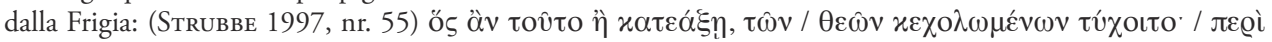

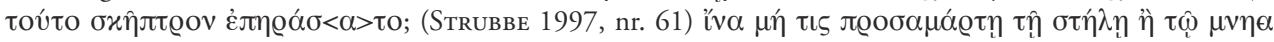


qui rappresenta un simbolo della potenza del dio, spesso denominato "re del villaggio" nelle acclamazioni che si trovano all'inizio di queste iscrizioni, alle quali si è accennato precedentemente. Questo rituale, accompagnato da preghiere e da altri atti cultuali e forse condotto da un sacerdote, sembra servisse per prendere contatto con la divinità, presentandogli la propria richiesta. Non possiamo neppure escludere, come potrebbe suggerire un confronto con le preghiere per la giustizia, che nell'ambito di questa azione rituale avvenisse anche la consacrazione dell'oggetto rubato al dio, di modo che questi si sentisse responsabile per il suo recupero. Il nostro testo non lascia capire se la vendita del mantello in questione dovesse essere fatta dal ladro, dal legittimo proprietario o dai sacerdoti del tempio. Caratteristico è ad ogni caso il desiderio della divinità a che le sue gesta tramite l'iscrizione venissero lodate e fatte conoscere agli altri fedeli, di modo che questi non sfidassero l'autorità ed il potere del dio (comunicazione religiosa orizzontale). L'iscrizione fa in modo che il potere (dynamis) o meglio l'attiva presenza del dio nel territorio dove sorge il santuario sia a tutti enarges ed epiphanestate.

Dalla Lidia di epoca imperiale facciamo ora cronologicamente un passo indietro a Cnido, dove nel santuario locale dedicato a Demetra ed a Kore sono state rinvenute diverse lamine plumbee di età tardo-ellenistica, contenenti una serie di preghiere per la giustizia. I fori apportati suggeriscono che queste lamine, nell'ambito di un rituale condotto dai sacerdoti del tempio, fossero state affisse sulle pareti del santuario, dove potevano essere lette (e commentate) dai visitatori di questo luogo sacro. Anche in questo caso la comunicazione religiosa orizzontale si rivela un fattore importante per la comprensione di queste iscrizioni; a riguardo si dovrebbe anche riflettere sull'effetto che questi testi potevano avere in persone appartenenti ad una piccola comunità cittadina, quale appunto Cnido, nella quale tutti si conoscevamo ed in cui, probabilmente, c'erano persone al corrente dei furti denunciati. Un'iscrizione che narra del furto di un mantello merita in questo contesto la nostra attenzione.

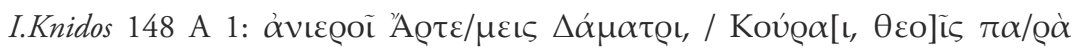

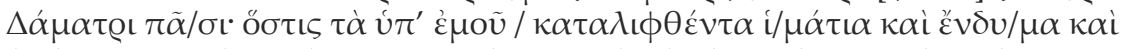

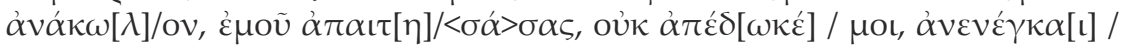

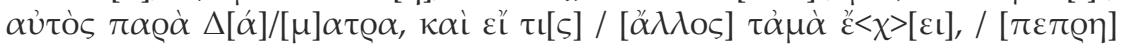

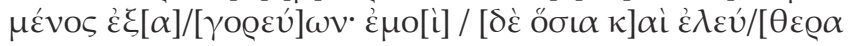

\section{B. 1}

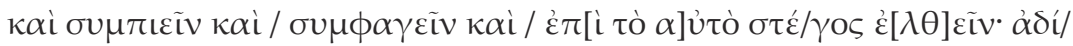
$\kappa \eta \mu \alpha \iota \gamma \alpha \dot{\varrho}, \delta \varepsilon \dot{\sigma} \sigma \pi[\mathrm{\iota}] / \nu \alpha \Delta \alpha \dot{\mu} \alpha \tau \varepsilon Q$.

Artemis consacra (questa tavoletta) a Demetra, a Kore ed a tutti quanti gli dei presso Demetra. Colui che non mi ha restituito i mantelli, gli indumenti e l'abito che dimenticai, pur avendone fatto richiesta, (prego affinché) costui riporti (questi indumenti) nel tempio di Demetra. Se qualcun altro ha in possesso un mio oggetto, questi faccia una confessione, bruciando. Per la mia persona sia invece tutto santo e libero, perché possa con tutti bere, mangiare e convenire nello stesso tetto. Infatti, o signora Demetra, a me è stata arrecata un'ingiustizia.

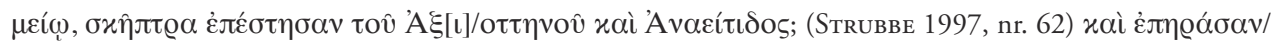

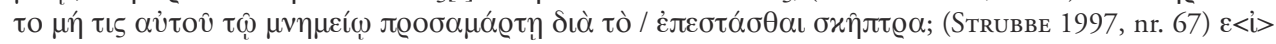

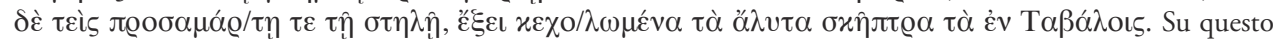
rituale rimando a GoRDON 2004b. 
Nonostante la distanza cronologica, questa epigrafe di Cnido si lascia confrontare con l'iscrizione confessionale prima analizzata ${ }^{59}$. Una donna di nome Artemis, alla quale non sono stati restituiti diversi capi di vestiario (tra cui dei mantelli), che essa aveva dimenticato o più probabilmente lasciati incustoditi (forse in un bagno pubblico), si rivolge alla dea più importante della città, affinché questa costringa il ladro (di cui non viene fatto il nome) a riportare la refurtiva nel tempio e a fare una confessione pubblica del suo reato. Il genitivo assoluto $\dot{\varepsilon} \mu o \tilde{v}$ $\dot{\alpha} \tau \alpha \iota \tau[\eta] /<\sigma \alpha \dot{\alpha}>\sigma \alpha \varsigma$ è atto a sottolineare che Artemis ha tentato di riavere indietro le sue cose e non essendovi riuscita, quale ultima istanza, si rivolge al potere della dea. L'espressione

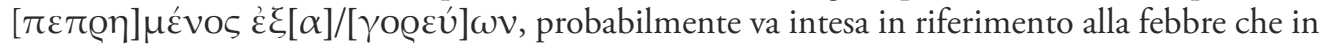
seguito alla punizione divina dovrebbe colpire il ladro ${ }^{60}$. Artemis può vivere tranquilla e continuare a bere e a mangiare con i suoi concittadini: lei è la persona che ha subito l'ingiustizia. Il testo di Cnido si lascia ben confrontare con quello lidio non solo da un punto di vista tema-

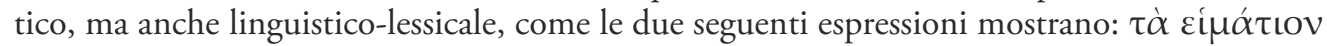

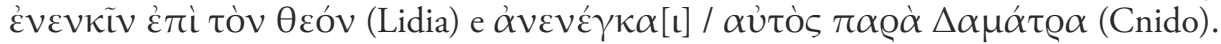

Le preghiere per la giustizia trovate in Inghilterra nei santuari di Uley ${ }^{61}$ e della dea Sulis ${ }^{62}$ a Bath offrono, in maniera analoga, un ricco materiale di paragone. In questi santuari è stato rinvenuto un gran numero di testi, apposti su lamine plumbee, contenenti questo genere di preghiera. Persone alle quali è stato sottratto un indumento (un abito o anche dei sandali) si rivolgono alla divinità del luogo, chiedendo non solo la restituzione del mal tolto, ma anche che il ladro venga tremendamente punito e faccia una confessione del reato nel santuario. Mi limito ad analizzare due testi provenienti dai due suddetti santuari, in cui si fa menzione di ladri di vestiti.

AE 1979, 384=Kropp 2008, 03.22.0363: Commonitorium deo / Mercurio a Satur/nina muliere de lint $<e=I>$ almine quod amisit ut il/le qui hoc circumvenit non / ante laxetur nis $\{s\}$ i quando / res $\{s\}$ s(upra) dictas ad fanum $\{s\}$ s(upra) dicltum attulerit si vir si mullier si servus si liber // Deo $\{s\}$ s(upra) dicto tertiam / partem donat ita ut / ex\{s\}igat istas res quae s(upra) s(crip)ta(e) sunt / ac \{a\} qua<s=E> per(didi)t deo Silvano / tertia pars donatur ita ut / hoc ex\{s\}igat si vir si femina si serv/us si liber [3]E[3] tat

Un memorandum al dio Mercurio da parte della donna Saturnina in relazione al vestito di lino che essa perse. (Saturnina si augura) che colui che le ha sottratto l'abito non trovi pace, sino a quando questi non riporti le cose sopraddette al sopraddetto tempio, sia che si tratti di un uomo, di una donna, di uno schiavo o di una persona libera. Al dio sopraddetto sia donata la terza parte (degli indumenti), affinché il dio esiga le cose sopra scritte, che Saturnina perse. Al dio Silvano sia donata la terza parte, affinché questi ne esiga (la restituzione), sia che si tratti di un uomo, di una donna, di uno schiavo o di una persona libera.

59 Un confronto tra le iscrizioni di Cnido e le iscrizioni confessionale era già stato fatto da VerSNEL 1991, 81-85; su questi testi da ultimo con bibliografia BERTI 2017, 81-86.

60 Cfr. VersNel 1994.

61 Su questo santuario cfr. WOOWARD-Leach 1993.

62 Su questo complesso sacro cfr. Cunlife-Davenport 1985; i testi epigrafici sono stati pubblicati in Tomlin 1988. In generale sui testi rinvenuti in Inghilterra Kiernan 2004.

63 Su questo testo cfr. TomLin 2010, 246-247 con annotazioni al testo. 
Tomlin 1988, 122-123, Nr. 10=AE 1982, 660=Kropp 2008, 03.02.10: Docilianus $/$ Bruceri / deae Sanctissim (a)e / Suli / devoveo eum [q] ui / carac $<a=E>$ Ilam meam / involaverit si / vir si femina si / servus si liber / ut [i e] um dea Sulis / maximo let $<0=U M>$ / [a]digat nec ei so/mnum permit//tat nec natos nec / nascentes do/[ne]c caracallam / meam ad tem/plum sui(!) numi/nis per[t]ulerit.

Io, Dociliano, figlio di Brucero, dedico alla santissima dea Sulis colui che ha rubato il mio mantello, sia che si tratti di un uomo, di una donna, di uno schiavo o di una persona libera. Questo perché la dea Sulis possa punirlo con la morte più dura, non gli permetta alcun sonno e di avere figlio ora ed in futuro, sino a quando questi non abbia riportato il mio mantello al tempio della sua divinità.

Entrambi i testi, scritti da uno specialista religioso, da identificarsi probabilmente con uno dei sacerdoti del tempio, si riferiscono a fatti di vita quotidiana. Una donna, Saturnina, ed un uomo di nome Dociliano, si rivolgono al loro dio chiedendo non solo il recupero del mantello rubato (che in un certo senso sembra passare in secondo piano), ma soprattutto la punizione del ladro. Come nei casi analizzati in precedenza, la punizione richiesta può sembrare esagerata, rispetto al crimine perpetrato, ma questi testi consentono di percepire le emozioni delle vittime. Essi ricordano i sentimenti di una persona alla quale è stato sottratto qualcosa di personale e che di primo impatto augura il peggio possibile al ladro, a prescindere dal valore dell'oggetto rubato. L'aspetto fortemente emozionale e soggettivo di questo testi è stato in anni recenti ben indagato da Angelos Chaniotis e Martin Dreher, i quali hanno sottolineato l'importanza di questo "elemento patetico" nelle pratiche di comunicazione religiosa nell'ambito di queste comunità di villaggio ${ }^{64}$. Proprio questo elemento, come in breve vedremo, può forse aiutarci a comprendere meglio la tradizione dei cani di Adrano.

\section{Adrano e le emozioni}

Torniamo ora al dio Adrano. Usando l'emotività come chiave di lettura, che può applicarsi anche alla sfera divina, poiché spesso nell'ambito della regione greca e romana le azioni degli dei sono guidate da emozioni come rabbia e pietà, il fatto che i ladri di mantelli venissero quasi sbranati dai cani indica il forte risentimento del dio nei confronti di persone che si sono comportate in maniera indegna nel suo santuario, macchiandosi di un crimine abbietto ${ }^{65}$. Un confronto con i documenti epigrafici sopra presi in considerazione permette di fare un ulteriore passo in avanti. Il dio, che dall'alto vede, sa e conosce chi ha peccato, non viene in questo caso invocato dal fedele derubato, ma nella sua funzione normativa agisce subito ed in maniera efficace, ristabilendo il giusto ${ }^{66}$. Da questo punto di vista, Adrano può, a mio avviso, essere ancor più accostato alle divinità adorate nei santuari rurali della Frigia e della Lidia, che venivano viste, come recita il

64 Chaniotis 2012; Dreher 2010; ID. 2012.

65 Sull'argomento segnalo i diversi contributi pubblicati nel dossier "Les dieux en (ou sans) émotion. Perspective comparatiste", apparso nel nr. 4 (2010) della rivista "Mythos".

66 Sul tema dell'onniscienza divina rimando al classico studio di Pettazzoni 1955, 208-239 (sulla Grecia), 240 258 (su Roma). Tra le tante testimonianze raccolte dallo studioso possiamo ricordare l'inizio del trattato plutar-

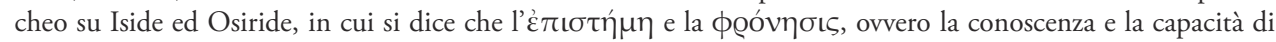
riflettere, rappresentano gli elementi costitutivi del divino ( $\tau$ ò $\theta \varepsilon \tilde{c} \mathbf{o v}$ ). 


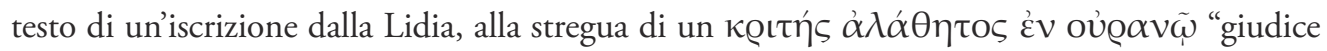
celeste infallibile" ${ }^{67}$. Questi racconti, o meglio queste tradizioni locali del santuario, che sicuramente circolavano in forma orale e che forse erano esposte in forma scritta nel santuario stesso, avevano probabilmente l'effetto di provocare timore nei confronti del potere del dio. Le persone che frequentavano questo luogo dovevano pensarci due volte prima di calcare il suolo sacro del santuario, essendosi macchiate di un crimine, ben sapendo quanto terribile poteva essere la punizione del dio. Come in Frigia, in Lidia ed in molti altri luoghi nell'Impero Romano, anche in questo caso la religione sembra assumere il ruolo di regolazione sociale, facendo in modo che le persone rispettassero le norme stabilite, astenendosi dal commettere reati. La giustizia divina, diretta, infallibile ed efficace, sembra porsi come valida alternativa a quella umana. Va inoltre posto in rilievo che le divinità menzionate nelle iscrizioni confessionali ed in diversi casi anche quelle invocate nelle preghiere per la giustizia da diversi punti di vista possono essere considerate come appartenenti alla suddetta categoria delle "Schutzgottheiten". Nel caso delle iscrizioni della Lidia e della Frigia, questi "dei del villaggio" venivano equiparati a dei re, che regnavano sulla comunità, proteggendola ed assicurandosi che leggi e norme morali del ben vivere non venissero violate. Da questo punto di vista la figura di Adrano è ben accostabile alle divinità menzionate in questi testi, e la sua attività quale "giudice punitore" risulta più comprensibile.

\section{Il santuario di Efesto ad Etna}

L'elemento che più colpisce nel culto di Efesto, nel santuario di Aitna-Inessa, è l'esercizio di una forma di giustizia divina, che prende nuovamente forma attraverso l'azione punitiva dei cani. Va, inoltre, sottolineato che tutte le notizie tramandate a riguardo si riferiscono al periodo a partire dal IV sec. a.C., epoca in cui la cultura delle società indigene presenta in maniera consolidata forti tratti greci. Eliano (n.a. XI, 20) narra che nel santuario di Efesto nella città di Etna i cani aggrediscono e mordono quanti osino porre piede nell'area sacra del santuario dopo essersi macchiati le mani di azioni esecrabili. Questi animali allontanano pure quanti tentano di avvicinarsi al tempio, dopo aver commesso atti sessuali.

Una caratteristica dei luoghi sacri, come noto, è quella della loro inviolabilità da parte di persone colpevoli di crimini, che con la loro impurità possono macchiare la sacralità del luogo $^{68}$. Le cosiddette leggi sacre greche sono ricche di norme in tal senso ${ }^{69}$, imponendo ad esempio un certo numero di giorni di astinenza sessuale ${ }^{70}$, prima di varcare la soglia di un tempio,

67 Herrmann - Malay 2007, 75-76, nr. 51=SEG LVII, 1159.

68 Su questo aspetto rimando al documentato studio di CHANiotis 1997, con un'impressionante raccolta di testimonianze letterarie ed epigrafiche sul tema.

69 Sul carattere di questi testi cfr. GUARDUCCI 1978, 3-45; i testi di queste iscrizioni sono stati raccolti ed editi con note di commento nei tre volumi curati da SoKolowski (1955, 1962, 1969); un'edizione recente con traduzione e commento in Lupu 2009. Per una critica e revisione del concetto di "legge sacra" rimando a Parker 2004 e Carbon - Pirenne-Delforge 2017.

70 Sokolowski 1969, nr. 55 (da Atene - l'ingresso al santuario di Men è permesso solo a quanti, dopo aver avuto rapporti sessuali con una donna, abbiano fatto un bagno; una donna, che abbia avuto mestruazioni può entrare solo dopo sette giorni e dopo essersi fatta un bagno); Sokolowski, 1962, nr. 91,3 (da Lindos - un uomo può accedere al santuario dopo un periodo di astinenza sessuale di 41 giorni). Per altre testimonianze cfr. FeHrLe 1910, 25-29; PARKer 1983, 74-100; anche nelle iscrizioni confessionali abbiamo casi di punizione a causa di intemperanze sessuali (Petzl 1994, nr. 5, 36). 
o vietando l'ingresso a persone che sono state in contatto con un morto o a donne che hanno partorito da poco. In una famosa iscrizione di Filadelfia (Lidia) troviamo addirittura l'esplicito divieto per persone i cui pensieri non sono puri ed innocenti ${ }^{71}$. I cani, gli animali sacri del dio ed i suoi ministri, si assicurano non solo che il volere della divinità sia rispettato, ma difendono la purezza sacrale del santuario. Le cronache templari antiche dovevano essere ricche di racconti, spesso anche molto coloriti, relativi a persone allontanate dal santuario e punite in quanto, in uno stato di impurità, avevano calcato il suolo sacro del luogo. Prendiamo ora in analisi il testo relativo al santuario di Efesto ad Etna, quale tramandato da Eliano ${ }^{72}$.

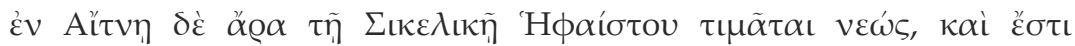

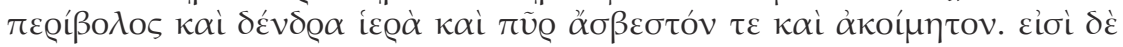

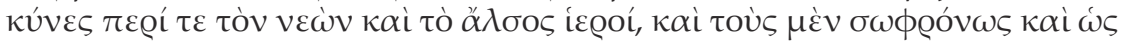

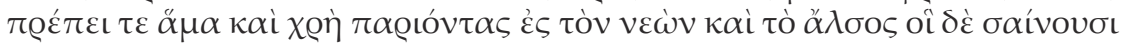

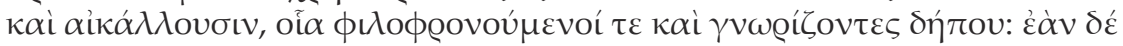

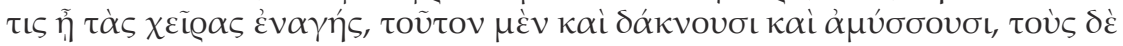

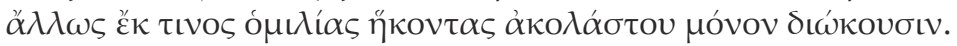

Nella città di Etna in Sicilia è oggetto di particolare culto il tempio dedicato a Efesto, in cui si trovano un recinto, degli alberi sacri e un fuoco inestinguibile e sempre acceso. Intorno al tempio e al bosco ci sono dei cani sacri. Se entrano nel tempio e nel bosco persone per bene, con un aspetto dignitoso e come si deve, i cani le accolgono in modo festoso e dimenando la coda, proprio come se le conoscessero e fossero ben disposti nei loro confronti; se invece entra qualcuno che ha le mani macchiate da un'azione esecrabile, lo mordono e lo aggrediscono violentemente. Si contentano invece di cacciare via quelli che hanno l'impudenza di venire, reduci da intemperanze sessuali. (Traduzione di Nicola Cusumano)

Dal testo apprendiamo che il santuario di Efesto, probabilmente, come detto, l'interpretatio

71 Si tratta di una lunga iscrizione datata tra il II ed il I sec. a.C., proveniente dal territorio di Filadelfia (Lidia), che contiene il regolamento cultuale di un culto privato di carattere misterico. Il testo, che per molti versi ricorda i precetti morali cristiani, sottolinea più volte l'importanza di avere pensieri puri ed intenzioni non malvagie quale requisito per essere ammessi al culto. Per un'edizione con commento e traduzione di questo testo cfr. PETZL 2007, nr. 1539; si vedano anche le osservazioni di CHAniotis 1997, 159-162 (che ne fornisce una traduzione in lingua tedesca) e da ultimo DE Hoz 2017. Un pensiero analogo sembra venisse espresso sull'inscrizione posta

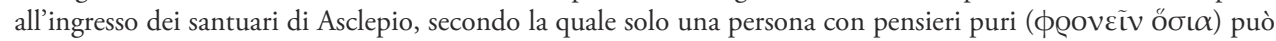
entrare nel tempio del dio; il testo di questa massima è stato tramandato da Porfirio (de abstinentia II, 19, 5) e da Clemente Alessandrino (Stromata V 1, 13, 3). In una aretalogia di Iside (Tотті 1985, nr. 11, 33-34) si legge che la dea aiuta volentieri quei mortali che sono di pensieri puri. In una lex sacra (SoKolowski 1969, nr. 82) da Rodi si trova la seguente ammonizione: «tu devi entrare puro e con puri pensieri dentro questo santuario». Per altri esempi rimando a CHAniotis 1997, 152-158, che è tornato recentemente sull'argomento in Chaniotis 2012b, il quale giustamente osserva (p. 133): «the development of the idea of the purity of the mind and its priority over the purity of the body was part of a more general process of fundamental changes in the perception of rituals in Classical and Hellenistic Greece. This process leads from the traditional view that ritual actions are effective when correctly performed to the idea that a ritual's efficacy depends (also) on the moral and other justifications of the individual who performs it.». Sull'argomento cfr. ora la documentata monografia di Petrovic - Petrovic 2016, con una ricca introduzione (pp. 1-37) contenente una storia degli studi.

72 Tra gli studi più recenti su questa tradizione cfr. Gagliano 2012, 306-308; Cusumano 2015, 73-93 con bibliografia precedente. 
greca di Adrano, che si trovava ad Etna, comprendeva un ampio recinto ed un bosco sacro, mentre all'interno del tempio ardeva perennemente un fuoco. Secondo questa tradizione, intorno al tempio ed al bosco vivevano i cani sacri del dio, i quali si curavano di difendere la purezza del luogo. La loro azione è volta al controllo di quanti entravano nello spazio sacro, i

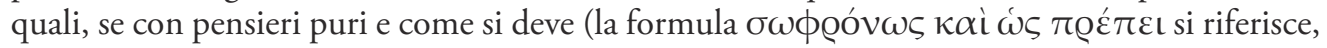
nell'ordine, alla purezza di pensieri ed all'adeguatezza dell'aspetto, probabilmente relazionata al portamento ed al modo di vestire) vengono accolti amichevolmente. Quanti invece si recano nel tempio con mani sacrileghe (l'espressione $\tau \hat{\alpha} \varsigma \chi \varepsilon \tilde{\imath} \varrho \alpha \varsigma \dot{\varepsilon} v \alpha \gamma \eta \dot{\zeta}$ si riferisce a chi si è macchiato di un'azione illecita), questi vengono presi a morsi in modo violento ${ }^{73}$. Va anche aggiunto che l'immagine delle mani macchiate, quale metafora per impurità, ricorre anche in diverse leggi sacre greche ${ }^{74}$ : questo fatto, non casuale, mostra l'adozione di un topos diffuso nell'ambito della letteratura templare antica nell'elaborazione di questa tradizione. I cani, probabilmente solo ringhiandovi contro, si limitano ad allontanare le persone che entrano nell'area templare dopo aver avuto dei rapporti sessuali. In questo contesto colpisce come i cani, tramiti del potere del dio in terra, riescano non solo a distinguere i puri dagli impuri, ma perseguano gli impuri in modo differente, a seconda della gravità della loro colpa. Quanti sono

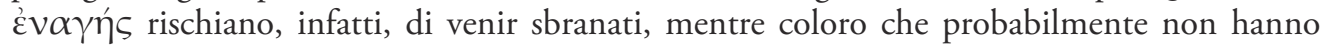
rispettato un certo lasso di tempo di astinenza sessuale, prima di entrare nel tempio, vengono semplicemente allontanati, senza danni fisici.

Eliano dichiara di aver attinto le notizie relative al ruolo dei cani nel culto di Adrano e dei Palici dall'opera di Ninfodoro, autore siracusano vissuto nella seconda metà del III

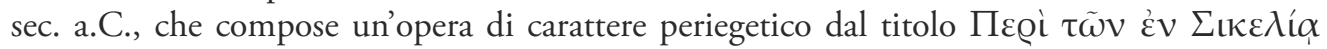
$\theta \alpha u \mu \alpha \zeta o \mu \varepsilon ́ v \omega v$ (FGrHist 572). Come Nicola Cusumano, a ragione, ha sottolineato, l'attività di questo intellettuale va considerata nel più ampio contesto della politica culturale di Ierone II, volta non solo al recupero ed alla valorizzazione del passato, ma anche finalizzata a ricostruire una linea di continuità con l'epoca dei Dinomenidi e dei Dionisii, come ad esempio mostra in maniera inequivocabile l'onomastica della nuova casa regnante. Va in ogni caso messo in rilievo che Ninfodoro nelle sue ricerche dovette molto probabilmente avvalersi di materiale d'archivio dei suddetti santuari, forse interrogando anche il clero locale. Il modo in cui in queste tradizioni si intravvede l'uso di elementi topici della mitologia greca lascia presumere che il processo di canonizzazione di queste tradizioni fosse da tempo avvenuto.

\section{Il santuario dei Palici}

Diodoro Siculo ed un autore tardoantico, Macrobio, rappresentano per noi le fonti più importante sul culto dei Palici nella città omonima ed in generale in Sicilia ${ }^{75}$. Il culto di queste

73 Parker 1983, 104-143; Chaniotis 1997, 148-151, che giustamente mette in rilievo che, seppure nel diritto greco fosse presa in considerazione la non intenzionalità di un delitto, quale attenuante, il colpevole era visto come impuro e macchiato dal miasma.

$74 \mathrm{Al}$ santuario di Zeus Kynthios e di Athena Kynthia a Delos possono aver accesso solo persone con un animo puro e con le mani pure (Sokolowski, 1962, nr. 59); stessa prescrizione si legge in una legge sacra da Lindos (SoкоLOWSKI 1969, nr. 139).

75 La città viene detta dalle fonti essere una fondazione di Ducezio; in realtà gli scavi condotti negli anni 60 sulla Rocchicella presso Mineo hanno portato alla luce i resti di strutture cittadine preesistenti con 
divinità autoctone si colloca in un paesaggio religioso molto particolare ${ }^{76}$, caratterizzato da una grotta, da un lago e da due crateri emananti vapori sulfurei ${ }^{77}$. Questo era il luogo in cui vivevano ed operavano i Palici, insieme ai loro fratelli, i Delli, considerati la personificazione dei due crateri lacustri, sopra menzionati. Questo santuario era a detta di Diodoro il più antico della Sicilia $^{78}$ ed il culto di queste divinità era di carattere ordalico. Secondo le fonti ${ }^{79}$, quanti erano stati accusati di furto o di un altro crimine si recavano nel santuario, dove presso i crateri prestavano un giuramento, tenendo un ramo fiorito in mano, indossando una veste senza cintura ed avendo sul capo una corona. Le regole rituali prescrivevano inoltre un certo periodo di digiuno e di astinenza sessuale per chi voleva sottoporsi a questa pratica. Dopo aver invocato il genio tutelare del luogo, si doveva porre per iscritto il giuramento su una tavoletta ${ }^{80}$, la quale veniva poi gettata nel cratere. Se la tavoletta restava a galla, era segno che la persona aveva giurato il vero, se affondava in questo caso si era commesso spergiuro $^{81}$. La punizione per tale atto era la morte ${ }^{82}$ o la cecità ${ }^{83}$, che colpiva immediatamente il reo colpevole di spergiuro $^{84}$. I Palici erano inoltre divinità oracolari ${ }^{85}$ e, forse per contaminazione con i Ca-

acropoli ed edifici di culto; pertanto quella di Ducezio sarebbe una rifondazione. cfr. ManisCaLCo - Mc-ConNELL 1997-1998, 173; sul sito cfr. anche le osservazioni di ADAMESTEANu 1962, 175-180; per una rassegna delle fonti e della bibliografia Di Stefano 1994, 280-282, s.v. Palike; Maniscalco - McConnell 2003; Cordano 2008; sugli aspetti storico-religiosi Cusumano 1990, 118-123; ID. 2006, 123-131; ID. 2015, 13-37. Copani 2008, identifica Palike con Trinakrie, centro quest' ultimo che secondo Antonio Franco (Franco 1999; ID. 2008, 165-170) sarebbe da identificarsi con Mendolito, i cui strati di distruzione, in effetti, sembrano coincidere col periodo in questione. Per gli scavi sul santuario rimando alla documentata monografia di ManisCalco 2008.

76 Il carattere autoctono di queste divinità veniva, ad esempio, evidenziato da Polemone (FHG III, $140 \mathrm{fr}$. 83=Ma-

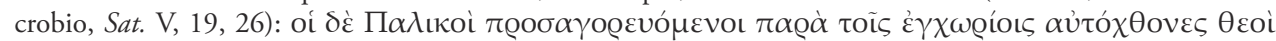

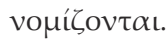

77 Si tratta, come giustamente messo in rilievo da Nicola Cusumano (Cusumano 2015, 19-22) di un locus inferus; osservazioni in proposito anche in Chirassi Colombo 2006, 224-227.

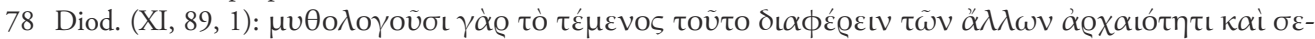

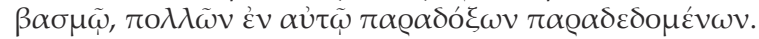

79 Le fonti principali sul giuramento ordalico nel santuario dei Palici sono il lemma Palike di Stefano di Bisanzio ed un passo dei mirabilia dello Pseudo-Aristotele (mir. ausc. 58). In considerazione dell'incredibile somiglianza dei due testi, è lecito supporre che si tratti di un estratto dall'opera di una fonte comune.

80 Non è da escludere che queste tavolette potessero essere scritte anche in lingua sicula, come afferma Nicola Cusumano (Cusumano 2006, 140-141).

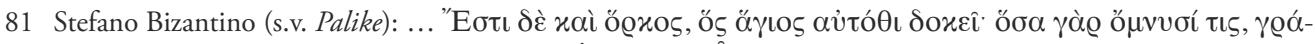

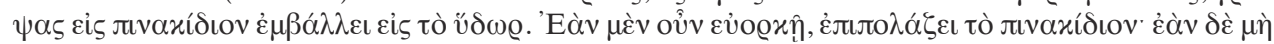

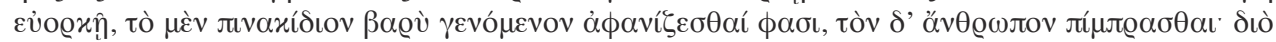

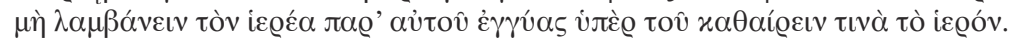

82 Nella tradizione tramandata dallo Pseudo-Aristotele e da Stefano di Bisanzio la morte avviene per combustione. Va rilevato che il verbo usato $\pi i ́ \mu \pi \varrho \alpha \sigma \theta \alpha \mathrm{s}$ si ritrova anche nelle preghiere per la giustizia e nelle defixiones, quale pena augurata al colpevole o alla persona sulla quale viene gettato il maleficio. Sull'uso di questo verbo in questi testi epigrafici rimando a VerSNEL 1994, il quale rileva che nei testi medici questo termine viene utilizzato in relazione agli effetti che la febbre alta ha sul corpo umano.

83 Cfr. Diodoro (XI, 89).

84 Il particolare della pena che arriva immediatamente accomuna tutti i testi che tramandano questa tradizione, come giustamente Nicola Cusumano (Cusumano 2006, 126, n. 19) sottolinea. Va rilevato che lo stesso motivo ricorre anche nelle preghiere per la giustizia, nelle preghiere per la vendetta e nei papiri magici, in cui il dio, dopo la lettura (ad alta voce) del testo rituale è invitato ad intervenire subito ed in maniera veloce.

85 Delle loro competenze oracolari ci informa un frammento dello storico Senagora (FGrHist 240 F 21=Macrobio, 
biri, vennero poi considerati come protettori dei naviganti ${ }^{86}$. Va aggiunto che il carattere oracolare di queste divinità, che come anche il diritto all'asylia di cui gode il santuario non verranno trattati approfonditamente in questo studio $^{87}$, ben si connette alla loro onniscienza, che, come anche per il loro genitore Adrano, permetteva loro di sapere se una persona stava mentendo o no nell'atto del giuramento. Quanto all'asylia, il fatto che in questo luogo potessero trovare rifugio schiavi maltrattati ingiustamente dai loro padroni ben si addice a delle divinità che, similmente al loro genitore, hanno un alto senso della giustizia, punendo lo spergiuro di ladri e malfattori.

Il processo di ellenizzazione del culto dei Palici si compie in primo luogo sul piano della memoria cultuale, creando, come detto prima, per questi dei una genealogia greca, secondo la quale essi sarebbero stati i figli di Zeus e della ninfa Talia ${ }^{88}$, che secondo un topos riscontrabile anche in altre tradizioni sarebbe stata costretta a nascondersi sottoterra per sfuggire all'ira di Era, gelosa ${ }^{89}$. Secondo un'altra versione del mito, essi sarebbero stati i figli di Efesto e della ninfa Etna; mentre secondo un'ulteriore variante più tarda essi sarebbero stati i figli di Efesto ${ }^{90}$. Va qui sottolineato come il processo di ellenizzazione del culto non abbia portato ad alcuna identificazione con una divinità greca (l'aspetto gemellare avrebbe potuto farli identificare con i Dioscuri, o anche con i Cabiri); la loro grecizzazione avviene tramite una manipolazione del mito, nonché tramite la creazione di un'etimologia greca del loro teonimo: essi sarebbero infatti stati coloro che "dalle tenebre ritornano di nuovo a questa luce" ( $\pi \dot{\alpha} \lambda \iota v \gamma \dot{\alpha} \varrho$ íkov $\sigma^{\prime} \dot{\varepsilon} \kappa$

Sat. V, 19, 30).

86 Questa tradizione risale a Varrone (Serv. Dan. Ad Aen. IX 581: Palicos nauticos deos appellat); su questa tradizione cfr. osservazioni in Cusumano 1990, 136 e Morawiecki 1995, 36-39, il quale sostiene che Varrone sarebbe stato a conoscenza della tradizione greca che faceva di Efesto il padre dei Palici, poi identificati con i Cabiri, in quanto Efesto era considerato il loro genitore. Non mi sento, tuttavia, di concordare con la proposta dello studioso, secondo cui l'identificazione di Adrano con Marte sarebbe avvenuta in epoca romana, dal momento che nessuna delle tradizioni che conosciamo identifica esplicitamente Adrano col dio della guerra; lo studioso afferma inoltre che la creazione del "singolo Palico", che troviamo menzionato in Virgilio (Aen. IX, 584-588) e nella relativa annotazione serviana, andrebbe datata in epoca romana.

87 Sul diritto all'asylia ci informa Diodoro (XI, 89, 6-8), a riguardo mi limito a rimandare a Cusumano 2006, 127-128; osservazioni anche in CARDETE Del Olmo 2007, 76-77.

88 Questa tradizione è attestata in un frammento di Eschilo (Fr. 27a Mette), trasmesso da Macrobio (Sat. V, 19,

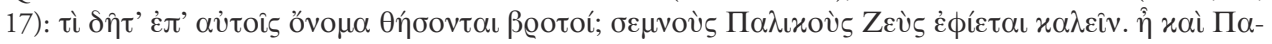

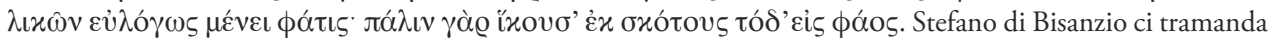
un frammento di Teofilo, autore di un'opera di carattere periegetico, in cui veniva citato un secondo frammento

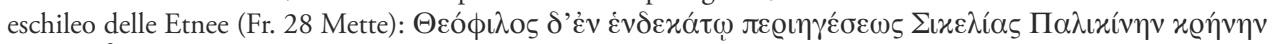

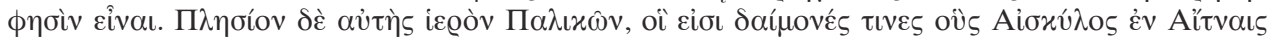

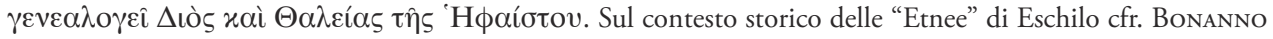
2010, 142-153; TOTARo 2011 con bibliografia precedente. A riguardo osservazioni in Cusumano 2006, 129131 e Copani 2007, 94-95, che vedono in questa grecizzazione genealogica dei Palici e nella creazione di questa paraetimologia un tentativo da parte dei Greci di appropriarsi di un culto indigeno. LuRAGHI 1994, 342-343 arriva a parlare di "assorbimento" dei Palici nel pantheon greco.

89 Il particolare che i gemelli fossero stati nascosti sottoterra è tramandato da Servio (Ad Aen. IX, 584: talis est fabula: Aetnam nympham (vel, ut quidam volunt, Thaliam) Juppiter cum vitasset, et fecisset gravidam, timens Junonem, secundum alios, ipsam puellam, Terrae commendavit, et illic enixa est. Secundum alios, partum eius, postea cum de

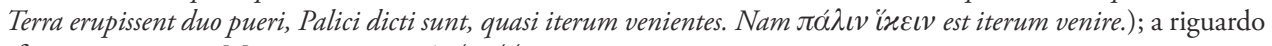
cfr. osservazioni in MORAwIECKI 1995, 43-44.

90 In generale per una rassegna delle fonti rimando a Rizzo 2012, 187-188. 


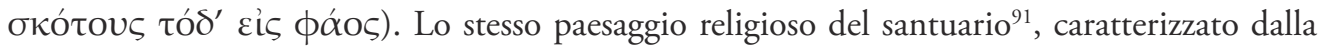
presenza di una grotta, di un laghetto (con vapori sulfurei) e di un bosco sacro, si sarebbe prestato alla traslazione di qualche mito antico ${ }^{92}$. Pensiamo, a mo' di esempio, alla facilità con la quale sulla base della presenza di una grotta in un monte e di un corso d'acqua molte città rivendicassero per il proprio territorio la nascita di Zeus ${ }^{93}$. Questo non avviene ed i Palici restano delle divinità indigene locali, assurgendo tuttavia al rango di figli di Zeus o di Efesto. In questa prospettiva di commistione del vecchio col nuovo concordo con l'interpretazione di Nicola Cusumano che vede nell'introduzione dell'uso della scrittura per il giuramento ordalico - sicuramente una pratica locale di sostrato - un elemento finalizzato «non a registrare o memorizzare passivamente l'oralità precedente, ma a stabilire un nuovo orizzonte di senso, e dunque a produrre una nuova forma di oralità ${ }^{94}$. In altre parole, la scrittura rappresenta quel quid che conferisce ad un rituale antico un carattere nuovo e vincolante.

Il rituale del giuramento ordalico nel santuario dei Palici ha molto in comune con le pratiche attestate nei santuari rurali dell'Asia Minore romana. Un nutrito numero di iscrizioni confessionali riguarda persone che hanno commesso spergiuro e che per questo sono state punite dal dio ${ }^{95}$. A titolo di esempio vorrei ricordare un'iscrizione nella quale il dio Men fece morire una donna, che sotto giuramento nel suo tempio aveva mentito, negando di aver ricevuto una data somma di denaro ${ }^{96}$. Va anche ricordato che nel mondo antico quello di prestare

91 Per una definizione di paesaggio religioso riporto le parole di Hubert Cancik (CANCIK 1985/86, 255): «sacred landscape is a constellation of natural phenomena constituted as a meaningful system by means of artificial and religious signs, by telling names or etiological stories fixed to certain places, and by rituals which actualize the space.». Tra i più recenti contributi sull'argomento segnalo ScheId - DE Polignac 2010, i quali, sottolineando l'assenza di un termine designante il paesaggio sacro in Greco ed in Latino, notano che (p. 431): «La notion de paysage religieux naît de la constatation que le culte et les rites n'existent qu'en tant qu'ils sont ancrés dans l'espace, que ce soit de manière stable ou pro visoire. Les temples, les sanctuaires, forment l'armature religieuse d'un territoire (de la même manière que les géographes parlent d'armature urbaine). Le paysage religieux procède ainsi d'une lecture symbolique de l'espace qui s'appuie elle- même sur les acquis de trente années de recherches en anthropologie religieuse.».

92 Sulle "sacred landscapes" in Sicilia rimando al documentato contributo di CARDETE 2010.

93 Si tratta di un tipo di paesaggio, che io ho proposto di definire "idaico" (cfr. Chiar 2017, 213-214). A riguardo va anche ricordato un famoso passo di Pausania (IV, 33, 1) nel quale il Periegeta in maniera alquanto ironica afferma che alla sua epoca è impossibile fare un elenco di tutti quelle località, che reclamavano per sé lo stato di luogo di nascita di Zeus. Lo stesso può dirsi per Apollo ed Artemide, i cui natali venivano contesi, ad esempio, da Efeso, Delo, Tebe e molte altre località del mondo greco. Sulle monete di Hierapolis in Frigia abbiamo la raffigurazione del ratto di Persefone ad opera di Ade: sulla base della presenza del famoso Plutonio, considerato come l'ingresso dell'Ade, questa città localizzava questo mito nel suo territorio. Un frammento dello storico Semos di Delo (FGrHist 396, F 20) informa che la Licia, Delo, l'Attica e la Beozia si contendevano il privilegio ai aver dato i natali ad Apollo. Sull'argomento cfr. anche il dettagliato studio di NolLè 2003, che analizza il materiale numismatico dell'Anatolia romana per ricostruire le numerose leggende locali sulla nascita di Zeus, localizzata ad esempio a Laodicea sul Lico, ad Aizanoi, ad Apamea etc.

94 Cusumano 2015, 25; in questo contesto possono essere citate anche le incisive parole di Simona Marchesini (MARCHESINi 1999, 175), in relazione alla valenza sacra della scrittura presso gli Italici: «suggellare il senso di sé con uno strumento che, nella sua funzione primaria, serve a fissare un messaggio altrimenti destinato a perdersi.....Ciò che sta scritto esige un vincolo estremo, sancisce quindi norme e codici.».

95 Ecco alcuni esempi: Petzl 1994, nr. 27, 34, 54, 58, 107. Sullo spergiuro nelle iscrizioni confessionali cfr. EGER 1939.

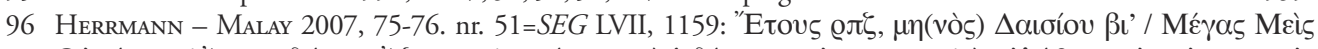

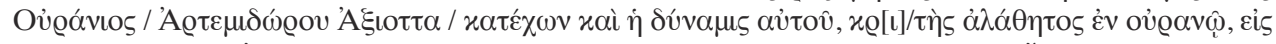

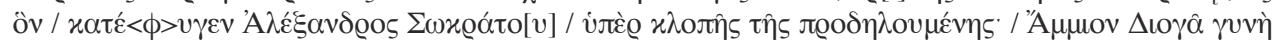


giuramento in un tempio in relazione a questioni giuridiche era un costume ben diffuso, che mostra come la giustizia divina fosse in molti casi avvertita come un'alternativa migliore a quella umana. Nelle petitiones dell'Egitto ellenistico-romano i giuramenti nei templi vengono addirittura menzionati quali argomento per indicare la mala fede di una persona ${ }^{97}$. Con una certa verosimiglianza, anche in virtù del confronto con le iscrizioni confessionali, possiamo presumere che tali contese avvenissero pubblicamente nel santuario e che gli esiti dei giudizi divini venissero scritti, conservati negli archivi del santuario e forse anche pubblicati su pietra o su tavolette di bronzo: tutte pratiche in cui viene usata la scrittura. L'introduzione della scrittura conferisce ad una pratica rituale antica un nuovo significato e valore. In questo contesto vorrei inoltre ricordare che le divinità tutelari (Schutzgottheiten), come Barbara Bratzeck ha sottolineato, sono solite essere invocate nei giuramenti. Il fatto che Palike, sotto Ducezio, divenga una polis sacra a queste divinità, può aver ulteriormente contribuito ad accentuare il legame tra giustizia e divino in questo luogo sacro.

\section{Contesto siculo, contesto frigio e contesto celtico: l'ellenizzazione ed i suoi effetti}

In questo contesto non intendo trattare il problema della ricostruibilità della religione indigena, tema sul quale, vale la pena ricordarlo, si espresse Angelo Brelich nel suo memorabile intervento del 1964, esprimendo, nella sostanza, il suo scetticismo circa la possibilità di usare come fonti le testimonianze di autori greci, che tramanderebbero comunque una visione greca dei fenomeni religiosi locali ${ }^{98}$. Un'interpretazione che si differenziava in maniera netta dai trends allora correnti, che vedevano nelle particolarità dei culti greci in Sicilia la testimonianza della continuità del sostrato locale, che i Greci, a seguito del contatto e della convivenza con le popolazioni indigene, avrebbero adottato ed adattato alla propria forma mentis ${ }^{99}$. Al problema di valutare come fonti per la religione indigena le testimonianze greche, si aggiunge la mancanza di testi scritti indigeni - fanno eccezione le brevi iscrizioni anelleniche, di non facile decifrazione $^{100}$, - ed il difficile uso degli artefatti archeologici ai fini di una ricostruzione delle pratiche cultuali $^{101}$. D'altronde va anche posto in rilievo, come ad esempio le aree sacre di Polizello ${ }^{102} \mathrm{e}$

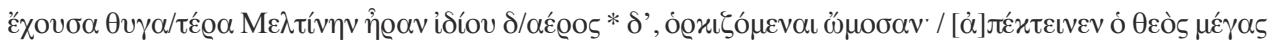
ó $\theta /[\varepsilon o ́ \varsigma . . . . U n$ altro interessante esempio è fornito dalla seguente iscrizione confessionale dalla Lidia, recentemente

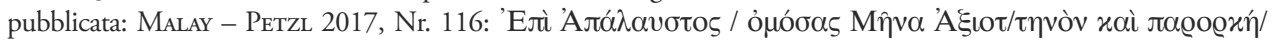

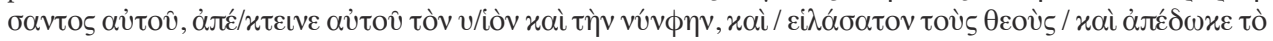

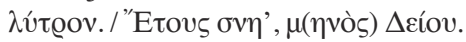

97 Ecco alcuni esempi Guéraud 1931, nr. 26, 5 -6 (giuramento prestato presso l'Arsinoeion); Guéraud 1931, nr. 47, 5-6 (giuramento prestato presso il tempio di Atena).

98 Brelich 1964-1965, su cui cfr. le importanti osservazioni di Cusumano 2005a; ID. 2005 b.

99 Questa impostazione di fondo caratterizzava, ad esempio, l'approccio di Biagio Pace alla cultura materiale dei popoli indigeni così come la ricostruzione fatta da Emanuele Ciaceri (CiaCERI 1911) dei culti e delle credenze (sull'opera di questo studioso cfr. Giammellaro 2008).

$100 \mathrm{~A}$ riguardo mi limito a rimandare al sempre valido contributo di Agostiniani 1988-1989; cfr. anche il più recente Agostiniani 2012 con bibliografia.

$101 \mathrm{Va}$ anche ricordato che solo a partire dalla Seconda Età del Ferro si hanno dati certi sulla strutturazione delle aree sacre, mentre per il periodo protostorico non si esclude che in alcuni edifici pubblici la funzione politica si affiancasse a quella sacrale. Per una sintesi cfr. Albanese Procelli 2003, 211-218; ead. 2006, 45-56.

102 ÖHLINGER 2015, 62-75, con bibliografia precedente. 
di Sabucina ${ }^{103}$ testimoniano, che proprio gli artefatti archeologici (modellini fittili di sacelli da Sabucina; statuette bronzee di offerenti, da Polizzello etc.) mostrano una forte influenza ellenica per quello che riguarda i media della comunicazione religiosa. Persino la stessa rappresentazione iconica della divinità in ambiente indigeno appare essere dovuta al contatto con i Greci, come testimonia la statua fittile di divinità femminile da Grammichele, di chiara ispirazione greca $^{104}$. A riguardo va segnalata la recente monografia di Birgit Öhlinger, che usa materiali archeologici per ricostruire da un punto di vista emico le pratiche rituali delle popolazioni indigene dell'entroterra nel periodo arcaico (VIII-V sec. a.C. $)^{105}$, sottolineando l'importanza dell'impatto con i Greci per una nuova definizione delle pratiche religiose locali ${ }^{106}$. È, infatti, un dato di fatto che solo a partire dalla Seconda Età del Ferro (VII sec. a.C.) in Sicilia si possiedono chiare testimonianze in relazione alla strutturazione delle aree sacre e che tale "riassetto del sacro" sia dovuto al contatto con i "nuovi arrivati"107. Fenomeni di convivenza, di mistione etnica e di ibridismo culturale nei territori coloniali - e la Sicilia da questo punto di vista non rappresenta certo un'eccezione ${ }^{108}$ - sono da tempo noti ed ampiamente studiati. Proprio per tal motivo, seppure con dovuta prudenza e prestando attenzione ai differenti contesti, possiamo evincere dalle testimonianze greche - siano esse di carattere archeologico, epigrafico o letterario - importanti elementi per la ricostruzione ed una migliore comprensione del mondo religioso indigeno. Circoscrivere il campo d'indagine alle sole evidenze archeologiche, oltreché essere riduttivo, può permettere (nel migliore dei casi) di conoscere una pratica cultuale solo nel suo aspetto materiale (e questo anche solo parzialmente), mentre gli aspetti legati alla sfera del mito, delle credenze etc. sfuggono ${ }^{109}$.

Tornando al tema centrale di questo studio, occorre ricordare che le tradizioni analizzate sui cani di Adrano e sui ladri di mantello si riferiscono ad un contesto non greco, ma siculo. Il

103 ÖHLINGER 2015, 76-85, con bibliografia precedente.

104 Albanese Procelli 2003, 271: «La rozza fattura, certamente indigena, tradisce l'ispirazione a modelli greci del periodo, reinterpretati con il gusto e le possibilità di tecniche locali.»; EAD. 2006, 56, dove viene sottolineato che le prime rappresentazioni iconografiche di divinità femminili in ambito indigeno non si datano prima del VI sec. a.C. e sono contemporanee alla diffusione del modello planimetrico greco dell'oikos bipartito.

105 Öhlinger 2015. La studiosa analizza 16 "Fallbeispiele".

106 Sulle culture e sui popoli anellenici della Sicilia, comunemente chiamati seguendo la tradizione letteraria (soprattutto quella tucididea) Sicani, Siculi ed Elimi, cfr. gli imprescindibili studi di Rosa Maria Albanese Procelli (Albanese Procelli i999; Ead. 2003; Ead. 2006; ead. 2010); un'ottima sintesi in La Rosa 1989 (ripreso poi in La Rosa 1996) ed in SPATAFora 2014; da ultimo cfr. la sopra citata monografia di ÖHLINGER 2015. Seppure invecchiati, per la ricchezza dei dati offerti, vanno citati PACE 1935-1949 e Bernabò Brea 1958. Per una sintesi storica cfr. Franco 2008, 71-105; Miccichè 2011. Sulle tradizioni mitiche rimando alle documentate monografie di Cusumano 1994 e Sammartano 1998.

107 Uno sguardo d'insieme in Albanese Procelli 2006, 56-66. La studiosa non esclude che i Greci avessero utilizzato le aree sacre quale luoghi di incontro e di scambio con le popolazioni locali.

108 Qui mi limito a rimandare alle recenti osservazioni di Carmine Ampolo (Ampolo 2012), che fornisce un'utile sintesi sul materiale epigrafico ed archeologico. Un paradigmatico caso di presenza di una comunità greca in un insediamento indigeno è quello di Monte Iato (cfr. la sintesi di IsLER 2012); i contesti funerari attestano ugualmente fenomeni di commistione e convivenza, per cui non sempre risulta possibile separare l'elemento indigeno da quello ellenico o fenicio (cfr. le sintesi di Albanese Procelli 2010; Spatafora 2010; Ead. 2012; Ead. 2014).

109 A riguardo riporto le parole di Rosa Maria Albanese Procelli (Albanese Procelli 2006, 44): «assumendo come fonti documentarie esclusivamente dati relativi alla cultura materiale, potremo avere un'idea delle pratiche di culto, cioè dei comportamenti esteriori e fattuali, e non certo delle credenze, la cui sfera involge fenomeni di natura emotiva e aspetti orali, coreografici e mitopoietici (nonché gestuali o relativi alla decorazione del corpo), difficilmente percepibili solo attraverso indicatori archeologici.». 
centro di Adrano venne, come detto in precedenza, fondato su iniziativa del tiranno di Siracusa, Dionisio il Vecchio, alle pendici dell'Etna, e destinato ad essere abitato dai Siculi. Prima ancora, nel 453 a.C., come Diodoro racconta (XI, 88, 6), Ducezio, dopo aver raccolto tutte le poleis sicule ad eccezione di Iblea in una confederazione, fondò presso il santuario dei Palici la città omonima, Palice ${ }^{110}$. Il confronto con i documenti dall'Asia Minore e dall'Inghilterra si fa in questo senso più interessante. La romanizzazione e, nel caso della Lidia e della Frigia, l'ellenizzazione non hanno comportato una cancellazione ed un oblio del sostrato religioso locale. L'interpretatio delle divinità indigene, come è stato sottolineato, ad una analisi più attenta si rivela spesso superficiale e riguarda in primo luogo il teonimo ${ }^{111}$. Come in particolare Jörg Rüpke in diversi contributi ha evidenziato ${ }^{112}$, la romanizzazione come anche l'ellenizzazione $e^{113}$ hanno comportato in primo luogo l'introduzione di nuovi media di comunicazione religiosa, primo fra tutti quello della scrittura, insieme alla diffusione di nuove pratiche religiose, che vanno a mischiarsi a quelle locali. Il rituale della confessione pubblica, nel caso della Frigia e della Lidia, risale sicuramente al sostrato locale, essendo ad esempio attestato già nei documenti ittitit $^{114}$. Come detto in precedenza, questo rituale è inoltre documentato anche in altri culti quali quello di Iside, della Dea Siria, nonché nelle pratiche religiose di diversi santuari di area celtica. Chiedersi se l'origine di questa pratica risalga ad un comune sostrato è forse chimerico e richiederebbe uno studio comparato ad esempio della religione ittita con quella celtica. Il punto più importante è che noi siamo a conoscenza di questa pratica grazie al fatto che la scrittura venne usata nei santuari rurali della Frigia e della Lidia per mettere su pietra le confessioni delle

110 Sulla synteleia di Ducezio mi limito a rimandare a Franco 2008, 147-158 con ampi riferimenti bibliografici; sull'uso di questo termine politico nell'opera diodorea cfr. Cusumano 1996.

111 A riguardo rimando alle osservazioni di CHiaI - KUnST - HäUssler 2012 ed ai contributi in Bricault-Bonnet 2013; Bonnet - Pirenne-Delforge - Pironti 2016.

112 RÜPKE 2010, ID. 2011; sull'argomento cfr. HAEUSSLER 2013, 27-73 con un'esaustiva bibliografia. La complessità del processo di romanizzazione viene riassunta da Ralph Häussler nel modo seguente (HaEussLer 2013, 18): «People were incorporated into the Imperium Romanum in such a way that their material culture, languages, cults, rituals and identities seemingly lost particularities, becoming almost indistinguishable from what could be found in Rome. But Rome did not create cultural homogenisation; instead, we must assume that local particularities persisted and continued to evolve gradually, comparable to the Roman East.».

113 BowERSOCK 1990, 1-28 fu ad esempio uno dei primi a sostenere che l'ellenizzazione, soprattutto grazie all'introduzione della scrittura, ebbe il merito di preservare il "locale" nelle diverse culture non-greche del mondo ellenistico (p. 7: «Hellenism was a language and culture in which peoples of the most diverse kind could participate. That is exactly what makes it remarkable. (...) It was a medium not necessarily antithetical to local or indigenous traditions. On the contrary, it provided a new and more eloquent way of giving voice to them»). Questa sua interpretazione ha trovato ampio consenso presso molti studiosi (cfr. ad esempio KAISER 2006, il quale nota (p. 29): «However, it has been convincingly argued that 'Hellenism' should not be viewed automatically as opposed to indigenous traditions: once transmitted to the Near East, Graeco-Roman cultural facets could come to serve as a medium by which a local culture could find renewed expression»). Sul ruolo del "particolare" e del "locale" nella vita religiosa del Vicino Oriente in epoca ellenistico-romana rimando ai diversi contributi in KAISER 2008. Del parere opposto è invece Millar 1987, 162-164, per quale l'ellenizzazione prima ed il dominio romano poi avrebbero condotto alla cancellazione ed all'oblio del "locale" (p. 162: «Everywhere except Mesopotamia, and of course Judaea, the combined effects of Hellenisation and Roman rule served in the medium term to suppress local identities»). Una discussione approfondita del concetto di ellenizzazione con un'ampia bibliografia si trova in StaVRianopoulou 2013 e BonNet 2014, 19-23; per chiarezza espositiva cfr. anche lo stimolante articolo di John $\mathrm{Ma}$ (MA 2008), che applica le categorie di "paradigma" e di "paradosso" allo studio dei processi di ellenizzazione.

114 Pettazzoni 1936, 39-53; sull'argomento anche FurLani 1935. Possiamo ricordare che questo rituale è attestato anche nelle iscrizioni sud-arabiche (cfr. SIMA 1999). 
persone che con le loro mancanze avevano sfidato la potenza del dio. Il porre questi racconti su pietra, quale forma di comunicazione religiosa orizzontale, può essere senza dubbio considerato un elemento di ellenizzazione (o di romanizzazione). Lo stesso discorso vale per le preghiere per la giustizia. Sicuramente questo rituale era praticato in forma orale prima della conquista romana in Britannia, dove i santuari delle divinità celtiche dovevano sicuramente fungere da importanti poli di riferimento per gli insediamenti sparsi nel territorio: pregare il dio, invocandone l'intervento per ottenere giustizia, è una pratica comune in tutte le religioni. La romanizzazione ha fatto in modo che la scrittura fosse introdotta nell'ambito di questa pratica rituale e che queste preghiere venissero inoltrate per iscritto alla divinità, un po' come succedeva per le suppliche inoltrate per iscritto al governatore locale. Il fatto che questi testi venissero redatti per iscritto ed in latino non inficia affatto l'origine locale di questa pratica rituale.

Anche nel caso del contesto siculo, uno degli effetti più visibili dell'ellenizzazione fu sicuramente quello dell'introduzione dell'alfabeto greco, che ci ha permesso di conoscere (seppure in maniera superficiale ed incompleta) la lingua sicula, le cui testimonianze epigrafiche cessano intorno alla metà del V sec. a.C. ${ }^{115}$. Greco è anche il modo in cui Ducezio riunisce in una confederazione le città sicule ${ }^{116}$, per le quali Diodoro forse non a caso usa il termine poleis. Questa confederazione fa poi capo al santuario dei Palici, che, forse su modello greco, diviene un santuario federale, nel quale vengono redatti, deposti e conservati gli atti di questa federazione ${ }^{117}$. Questo tipo di confederazione si connette certamente con l'emergere di una consapevolezza etnica in funzione antiellenica, ma anche e soprattutto con un nuovo ordine mentale. Sicuramente nel santuario i rappresentanti delle città confederate, oltre a riunirsi, dovettero prestare anche un giuramento della cui validità dovevano farsi garanti le divinità del santuario. Questo aspetto di garanzia giuridica ben si confa col carattere di giudice che punisce i malfattori, quale testimoniato nelle tradizioni: un aspetto che viene alla luce e che conosciamo grazie all'uso della scrittura che venne fatto in questo contesto.

\section{Conclusioni}

Lo scopo di questo studio è stato quello di indagare le modalità in cui, a seguito del contatto dei Greci con le popolazioni indigene in Sicilia, nella sfera religiosa si introducano nuove forme di comunicazione: di queste l'uso della scrittura come medio di comunicazione religiosa (orizzontale e verticale) rappresenta forse l'innovazione più importante. A questi nuovi media siamo,

115 In generale sulla lingua sicula e sulle interferenze linguistiche col greco rimando a WiLLi 2008, 341-347 con bibliografia.

116 Questo discusso personaggio viene in maniera pregnante definito come «espressione di un'élite indigena culturalmente e militarmente evoluta, desiderosa di affermare i propri diritti e di sottrarsi all'egemonia delle colonie greche» (cfr. Simonetti Agostinetti 2012, 322). Su Ducezio e la sua importanza storica esiste una vasta bibliografia, in generale cfr. ADAMESTEANu 1962 (al quale si deve la pregnante frase "il momento di Ducezio"; lo studioso fu uno dei primo ad aver studiato il testo di Diodoro in relazione alle evidenze archeologiche); GaLvagno 1991 (sull'uso retorico della figura del condottiero siculo nell'ambito dell'opera diodorea); CARDETE DEL Olmo 2007 (con un'analisi del rapporto di Ducezio col santuario dei Palici); Simonetti AgostinetTi 2012 (con una buona sintesi storica degli avvenimenti); BelLino 2014 (che analizza gli aspetti militari). CopANi 2007; ID. 2008 sulle fondazioni di Trinakie e Palike; Franco 2008, 139-170. Da ultimo Cusumano 2015, 14-19 con bibliografia.

117 A riguardo rimando alle osservazioni di Cusumano 2015, 19-37; sul tema dei santuari federali greci rimando ai recenti contributi in Funke - HaAke 2013. 
inoltre, debitori della conoscenza della morfologia divina di Adrano e dei Palici. Le (poche) tradizioni conosciute concordano nel sottolineare l'antichità ( $\alpha \varrho \chi \alpha$ เó $\rceil\rceil \varsigma)$ dei culti di queste divinità, dai cui interventi (diretti o tramite i cani) si evince il modo in cui questi dei fossero considerati alla stregua di giudici divini, ai quali stava a cuore la purezza dei loro luoghi di culto, non tollerando che in questi entrassero malfattori o persone malvagie. Proprio questo loro carattere, estraneo alla mentalità greca, che, seppur con la dovuta cautela dovuta alla distanza cronologica e geografica, rende possibile fare un paragone con le iscrizioni confessionali e con le preghiere per la giustizia, permette anche di intravedere la vitalità con cui le credenze locali, seppure in una nuova veste (ed in primis in una nuova lingua) continuino a vivere. Come nelle comunità di villaggio della Frigia e della Lidia romana e come le presso le comunità celtiche romanizzate della Britannia, anche in questo caso la religione ed il potere divino sembrano porsi come un'alternativa efficace nei confronti della giustizia umana, o forse anche nei confronti della giustizia portata dai conquistatori greci e romani, quale forma di resistenza culturale. Si tratta di una situazione che per molti versi, a buon diritto, si potrebbe definire di prédroit ${ }^{118}$, la quale per sua natura ha poco di greco, ma che, paradossalmente, conosciamo grazie all'ellenizzazione di questi culti. Questo può forse anche in parte spiegare il motivo per cui l'identificazione di Adrano e dei Palici con una divinità greca sembra non essere riuscita del tutto. Anche nel caso del santuario di Efesto ad Etna, il carattere anellenico del culto doveva risultare (soprattutto ad un Greco) abbastanza evidente. Si tratta di una forma di resistenza che riguarda non solo le forme cultuali, ma, in questo caso, in primo luogo i teonimi. Concludendo, sarebbe interessante indagare il modo e le forme in cui, in seguito all'ellenizzazione ed alla romanizzazione della Sicilia, il "locale" continui non solo a vivere, ma in maniera consapevole ad essere utilizzato per far emergere la propria identità e diversità rispetto a chi è arrivato più tardi. Greg Wolf ha, ad esempio, mostrato che nella Gallia, dopo la conquista romana, sulla base soprattutto delle fonti documentarie si possono ricostruire due fasi di romanizzazione ${ }^{119}$. Durante la prima, che abbraccia il periodo augusteo e tutto quanto il I sec. d.C., abbiamo la formazione di una cultura mista, denominata gallo-romana, mentre nella seconda ha luogo una differenziazione locale di questa cultura gallo-romana, che mostra il "Weiterleben" delle particolarità regionali delle preesistenti culture celtiche. Proprio il persistere di questi particolarismi, che hanno, per altro, fatto in modo che nello studio della romanizzazione venissero introdotti termini e concetti moderni come "creolizzazione", "globalizzazione" etc. ${ }^{120}$, ci mostra l'importanza del "locale" per la determinazione della propria identità nel contesto dell'Impero Romano ${ }^{121}$.

Gian Franco Chiai Institut für Alte Geschichte Freie Universität Berlin Koser Str. 20 14195 Berlin gian.franco.chiai@fu-berlin.de

118 Su questo concetto, introdotto da Louis Gernet (Gernet 1948/49) cfr. TAdDei 2009 e Cusumano 2015, 67-68 con bibliografia.

119 Wolf 1998; lo stesso modello è stato applicato da Ralph Häussler (HaEussLer 2013) allo studio della romanizzazione del nord-ovest Italia.

120 Sui pericoli dell'uso di tali categorie moderne nello studio del mondo antico rinvio a TraINa 2006.

121 La valorizzazione del "locale" nello studio dell'evoluzione della cultura nelle diverse provincie dell'Impero Romano acquista sempre più importanza nella ricerca; su questo fenomeno segnalo un importante progetto di ricerca condotto da Hans Beck (http://www.hansbeck.org/local/). Un'ottima rassegna delle fonti letterarie, che mettono in luce l'importanza del "particolare" nella percezione della Sicilia nell'immaginario romano si trova in CHIRAssi CoLOMBo 2006. 


\section{Bibliografia}

\section{Adamesteanu 1962}

D. Adamesteanu, «L'ellenizzazione della Sicilia ed il momento di Ducezio", Kokalos 8 (1962), 167-198.

Agostiniani 1988-1989

L. Agostiniani, «I modi del contatto linguistico tra Greci e Indigeni nella Sicilia antica», Kokalos 34-35 (1988-1989), 167-208.

\section{Agostiniani 2009}

L. Agostiniani, «Le iscrizioni anelleniche del Museo di Adrano", in LAMAGNA 2009b, 115-118.

\section{Agostiniani 2012}

L. Agostiniani, «Alfabetizzazione della Sicilia pregreca", in Convivenze etniche, scontri e contatti di culture in Sicilia e Magna Grecia, Aristonothos. Scritti per il Mediterraneo antico, 7 (2012), 139-164.

\section{Albanese Procelli 1999}

R.M. Albanese Procelli, «Identità e confini etnico-culturali: la Sicilia centro-orientale», in Confini e frontiera nella Grecità d'Occidente. Atti del trentasettesimo convegno di studi sulla Magna Grecia (Taranto 3-6 ottobre 1997), Napoli 1999, 327-359.

\section{Albanese Procelli 2003}

R.M. Albanese Procelli, Sicani, Siculi, Elimi: forme di identità, modi di contatto e processi di trasformazione, Milano 2003.

\section{Albanese Procelli 2006}

R.M. Albanese Procelli, «Pratiche religiose in Sicilia tra Protostoria e Arcaismo», in P. Anello - G. Martorana - R. Sammartano (a cura di), Ethne e religioni nella Sicilia antica. Atti del convegno (Palermo, 6-7 dicembre 2000), Roma 2006, 43-70.

\section{Albanese Procelli 2009}

R.M. Albanese Procelli, «Il centro del Mendolito di Adrano: la produzione metallurgica», in G. Lamagna (ed.), Tra Etna e Simeto. La ricerca archeologica ad Adrano e nel suo territorio, Catania 2009, 105-114.

Albanese Procelli 2010

R.M. Albanese Procelli, «Presenze indigene in contesti coloniali sicelioti: sul problema degli indicatori archeologici», in H. Tréziny (éd.), Grecs et Indigènes de la Catalogne à la mer Noire, Actes des rencontres du programme européen Ramses 2 (2006-2008), Paris 2010, 501-508.

Ampolo 2012

C. Ampolo, "Compresenze di ethne e culture diverse nella Sicilia occidentale. Per una nuova prospettiva storica", in Convivenze etniche, scontri e contatti di culture in Sicilia e Magna Grecia, Aristonothos. Scritti per il Mediterraneo antico, 7 (2012), 15-57.

BELAYCHE 2006a

$\mathrm{N}$ Belayche, " $\mathrm{Au}(\mathrm{x})$ dieu(x) qui règne(nt) sur.....» Basileia divine et fonctionnement du polythéisme dans l'Anatolie impériale», in A. Virgout - X. Loriot - A. Berenger-Badel - B. Klein (éds.), Pouvoir et religion dans le monde romain. En hommage à Jean-Pierre Martin, Paris $2006,257-269$.

BeLAYCHe 2006b

N. Belayche, «Les steles dites de confession: une religiosité originale dans l'Anatolie impériale?», in L. De Blois - P. Funke - J. Hahn (eds.), The impact of imperial Rome on religions, ritual and religious life in the Roman Empire, Boston-Leiden 2006, 66-81.

BeLAYCHe 2007a

N. Belayche, "Rites et „croyances“ dans l'épigraphie religieuse de l'Anatolie impériale», in J. Scheid (éd.), Rites et croyances dans les religions du monde romain, Entretiens sur l'antiquité classique LIII, Genève 2007, 73-103.

BeLAYCHe 2012

N. Belayche, «Un châtiment en adviendra». Le malheur comme signe des dieux dans l'Anatolie impériale», in S. Georgoudi - R. Koch Piettre F. Schmidt (éds.), La Raison des signes. Présages, rites, destin dans les sociétés de la Méditerranée ancienne, Leiden-Boston 2012, 319-342.

Bellino 2014

V. Bellino, «L'esercito di Ducezio. Guerra e influenze culturali durante il periodo della synteleia», Studi Classici e Orientali LX (2014), 53-71. 
Bernabò Brea 1958

L. Bernabò Brea, La Sicilia prima dei Greci, Milano 1958.

BERTI 2017

I. Berti, Gerechte Götter?: Vorstellungen von göttlicher Vergeltung im Mythos und Kult des archaischen und klassischen Griechenlands, Heildelberg (Propylaeum-eBOOKS) 2017.

BјÖRCK 1938

G. Björck, Der Fluch des Christen Sabinus: Papyrus Upsaliensis 8, Uppsala 1938.

Bonanno 2010

D. Bonanno, Ierone il Dinomenide. Storia e rappresentazione, Supplementi a Kokalos 21, PisaRoma 2010.

Bonnet 2014

C. Bonnet, Les enfants de Cadmos. Le paysage religieux de la Phénicie hellénistique, Paris 2014.

BOWERSOCK 1990,

G. Bowersock, Hellenism in Late Antiquity, Ann Arbor 1990.

BRELICH 1949

A. Brelich, Die geheime Schutzgottheit von Rom, Zurich 1949.

BRELICH, 1964-1965

A. Brelich, "La religione greca in Sicilia», in ACISSA I, Kokalos X-XI (1964-1965), 35-54.

BRACKerTZ 1976

U. Brackertz, Zum Problem der Schutzgottheiten griechischer Städte, Berlin 1976.

BURKeRT 1994

W. Burkert, Vergeltung zwischen Ethologie und Ethik. Reflexe und Reflexionen in Texten und Mythologien des Altertums, Carl Friedrich von Siemens Stiftung, Reihe Themen 55, München 1994

Canciani 1981

F. Canciani, s.v. Adranos I, in LIMC, I, 1, Zürich-München 1981, coll. 229-230.

CANCIK 1985-1986

H. Cancik, «Rome as a Sacred Landscape. Varro and the End of Republican Religion in Rome», Visible Religion 4-5 (1985-1986), 250265.
Cantarella 1991

E. Cantarella, I supplizi capitali in Grecia e a Roma, Milano 1991.

Carbon - Pirenne - Delforge 2017

J.-M. Carbon - V. Pirenne-Delforge, «Codifying 'Sacred Laws' in Ancient Greece», in D. Jaillard - C. Nihan (eds.), Writing Laws in Antiquity. L'écriture du droit dans l'Antiquité, Wiesbaden 2017, 141-157.

Cardete del Olmo 2007

M.C. Cardete del Olmo, «El silencio de los oprimidos: el culto de los Paliki», Ilu. Revista de Ciencias de las Religiones 19 (2007), 67-84.

Cardete del Olmo 2010

M.C. Cardete del Olmo, Paisaje, identidad y religión: imágenes de la Sicilia antigua, Barcelona 2010.

\section{Cerchiai 2017}

L. Cerchiai, «Integrazione e ibridismi campani: Etruschi, Opici, Euboici tra VIII e VII sec. a.C.», in Ibridazione e integrazione in Magna Grecia. Forme modelli dinamiche. Atti del cinquantaquattresimo convegno di studi sulla $\mathrm{Ma}$ gna Grecia (Taranto 25-28 settembre 2014), Taranto 2017, 221-243.

\section{Chaniotis 1995}

A. Chaniotis, «Illness and cures in the Greek propitiatory inscriptions and dedications of Lydia and Phrygia», in H.F.J. Hormannshoff - Ph.J. van der Eijk - P.H. Schrijvers (eds.), Ancient Medicine in its Socio-Cultural Context. Papers Read at the Congress Held at Leiden University (13-15 April 1992), Amsterdam-Atlanta 1995, 323-344.

\section{Chaniotis 1997a}

A. Chaniotis, «Tempeljustiz im kaiserzeitlichen Kleinasien: Rechtliche Aspekte der Beichtinschriften", in G. Velissaropoulos-Karakostas (Hrsg.), Symposion 1995. Vorträge zur griechischen und hellenistischen Rechtsgeschichte, Köln 1997, 353-384.

\section{Chaniotis 1997b}

A. Chaniotis, «Reinheit des Körpers - Reinheit des Sinnes in den griechischen Kultgesetzen", J. Assmann - T. Sundermeier - H. Wrogemann (Hrsg.), Schuld, Gewissen und Person. Studien 
zur Geschichte des inneren Menschen, Gütersloher 143-179.

\section{Chaniotis 1998}

A. Chaniotis, "Willkomene Erdbeben», in E. Olshausen - H. Sonnabend (Hrsg.), Naturkatastrophen in der antiken Welt, Stuttgarter Kolloquium zur historischen Geographie des Altertums 6, Stuttgart, 404-416.

\section{Chaniotis 2004}

A. Chaniotis, «Under the Watchful Eyes of the Gods: Aspects of Divine Justice in Hellenistic and Roman Asia Minor", in S. Colvin (ed.), The Greco-Roman East. Politics, Culture, Society, Yale Classical Studies 31, Cambridge 2004, $1-43$.

Chaniotis 2005

A. Chaniotis, War in the Hellenistic World, 2005 Oxford.

Chaniotis 2008

A. Chaniotis, «Konkurrenz und Profilierung von Kultgemeinden im Fest», in J. Rüpke (Hrsg.), Festrituale in der römischen Kaiserzeit, Tübingen 2008, 67-87.

Chaniotis 2009

A. Chaniotis, «Ritual Performances of Divine Justice. The Epigraphy of Confession, Atonement, and Exaltation in Roman Asia Minor», in H. Cotton (ed.), From Hellenism to Islam. Cultural and Linguistic Change in Roman Near East, Cambridge 2009, 115-153.

Chaniotis 2010

A. Chaniotis, «Megatheism. The search for the almighty god and the competition of cults", in S. Mitchell - P. Van Nuffelen (eds.), One God. Pagan Monotheism in the Roman Empire, Cambridge 2010, 112-140.

Chaniotis 2012a

A. Chaniotis, "Constructing the Fear of Gods. Epigraphic Evidence from Sanctuaries of Greece and Asia Minor", in A. Chaniotis (ed.), Unveiling Emotions. Sources and Methods for the Study of the Emotions in the Greek World, HABES 52, Stuttgart 2012, 205-234.

Chaniotis 2012b

A. Chaniotis, «Greek Ritual Purity: From Au- tomatisms to Moral Distinctions", in P. Roesch - U. Simon (eds.), How Purity is made, Wiesbaden 2012, 123-139.

Chiai 2008

G.F. Chiai, «Il villaggio ed il suo dio: considerazioni sulla concorrenza religiosa nelle comunità rurali dell'Asia Minore in epoca romana", $M y$ thos 1 (2006-2007) 2008, 137-163.

Chiar 2009a

G.F. Chiai, «Die Ortsgebundenheit des Religiösen: Das Paradigma der ländlichen Heiligtümer Phrygiens in der Kaiserzeit», in C. Auffahrt (Hrsg.), Religion auf dem Lande, Stuttgart 2009, 133-160.

Chiar 2009b

G.F. Chiai, «Königliche Götter und gehorsame Untertanen im Kleinasien der Kaiserzeit: Zur Funktion der Machtepitheta in religiöser Kommunikation», in J. Rüpke (Hrsg.), Die Religion des Imperium Romanum. Koiné und Konfrontation, Tübingen, 219-247.

Chiai 2010

G.F. Chiai, «Perché un dio è potente? Considerazioni sull'enoteismo e sulla costruzione del divino in Asia Minore», Studi e Materiali di Storia delle Religioni 76 (2010), 187-230.

Chiar 2012

G.F. Chiai, «Die Götter und ihr Territorium: Münzen als Quellen zur interpretatio im kaiserzeitlichen Phrygien", Mediterraneo Antico 15 (2012), 51-70.

Chiar 2013

G.F. Chiai, «Die Ortsgebundenheit des Göttlichen bei Macrobius», in A. Fürst - L. Ahmed - C. Gers-Uphaus - S. Klug (Hrsg.), Monotheistische Denkfiguren in der Spätantike, Studien und Texte zu Antike und Christentum 81, Tübingen 2013, 215-242.

Chiai 2017

G.F. Chiai, Troia, la Troade ed il Nord Egeo nelle tradizioni mitiche greche. Contributo alla ricostruzione della geografia mitica di una regione nella memoria culturale greca, Mittelmeerstudien 16, Paderborn 2017. 
Chiai - HäUssler - Kunst 2012

G.F. Chiai - R. Häußler - C. Kunst, «Interpretatio Graeca, Romana und Indigena: Religiöse Kommunikation zwischen Globalisierung und Partikularisierung», Mediterraneo Antico 15, (2012), 51-70.

\section{Chirassi Colombo 2006}

I. Chirassi Colombo, «La Sicilia e l'immaginario romano", in P. Anello - G. Martorana - R. Sammartano (a cura di), Atti del Convegno Internazionale Ethne e religioni nella Sicilia antica (Palermo 5-6 dicembre 2000), Supplementi a Kokalos 18, Roma 2006, 217-249.

Ciaceri 1911

E. Ciaceri, Culti e miti nella storia dell'antica Sicilia, Catania 1911.

Copani 2007

F. Copani, «Il mito di Trinaco e la propaganda di Ducezio", Parola del Passato 62 (2007), 81-98

\section{Copani 2008}

F. Copani, «L'enigmatica città di Trinakìe», in T. Alfieri Tonini (a cura di), Mythoi siciliani in Diodoro. Atti del seminario di studi, Aristonothos 2 (2008), 51-69.

\section{Cordano 2008}

F. Cordano, «Il santuario dei Palikoí», in T. Alfieri Tonini (a cura di), Mythoi siciliani in Diodoro. Atti del seminario di studi, Aristonothos 2 (2008), 41-49.

\section{Cunliffe-Davenport 1985}

B. Cunliffe - P. Davenport (eds.), The Temple of Sulis Minerva at Bath. Volume 1. The Site, Oxford 1985.

\section{Cusumano 1990}

N. Cusumano, "Ordalia e soteria nella Sicilia antica. I Palici», Mythos 2 (1990), 9-186.

\section{Cusumano 1992}

N. Cusumano, «I culti di Adranos ed Efesto. Religione, politica e acculturazione in Sicilia tra V e IV secolo", Kokalos 38 (1992), 151-189.

\section{Cusumano 1994}

N. Cusumano, Una terra splendida e facile da possedere. I Greci e la Sicilia. Supplementi a Kokalos 10, Roma 1994.

\section{Cusumano 1995}

N. Cusumano, «Sul lessico politico di Diodoro: synteleia», Kokalos 41 (1995), 151-189.

Cusumano 2004

N. Cusumano, «Animali, culti e interazioni etniche. I ladri di mantello ad Atene e Adrano tra droit e prédroit», Mythos 12, 2004/2005 (2006), 107-136.

\section{Cusumano 2005}

N. Cusumano, «Una storiografia a rovescio. Angelo Brelich e la religione greca in Sicilia», in M.G. Lancellotti - P. Xella (a cura di), Angelo Brelich e la storia delle religioni: problemi e prospettive (Atti del Convegno di Roma, 3-4 dicembre 2002), Verona 2005, 83-106.

Cusumano 2006

N. Cusumano, «I Siculi», in P. Anello - G. Martorana - R. Sammartano (a cura di), Atti del Convegno Internazionale Ethne e religioni nella Sicilia antica (Palermo 5-6 dicembre 2000), Supplementi a Kokalos 18, Roma 2006, 121-146.

\section{Cusumano 2015}

N. Cusumano, Adrano, Efesto e i Palici. Culti, interazioni e middle ground nella Sicilia antica, Caltanisetta - Roma 2015.

De Hoz 1999

M.P. de Hoz, Die lydischen Kulte im Lichte der griechischen Inschriften, AMS 36, Bonn 1999.

D'ANDRIa 2012

F. d'Andria, «Il santuario e la tomba dell'apostolo Filippo a Hierapolis di Frigia", Rendiconti della Pontificia Accademia Romana di Archeologia LXXXIV (2011-2012), 1-52.

De VIDo 2011

S. De Vido, «Timoleonte liberatore: appunti per una biografia», in M. Congiu - C. Micciché - S. Modeo (a cura di), Timoleonte e la Sicilia della seconda metà del IV sec. a.C., Atti del VII Convegno di studi, Caltanissetta 2011, 9-20.

De VIDo 2013

S. De Vido, Le guerre di Sicilia, Roma 2013.

DiCKIE 2002

M.W. Dickie, «Who Were Privileged to See the Gods?», Eranos 100 (2002), 109-127. 
Dignas 2003

B. Dignas, "Religious Centres in the Greek East. Worlds Apart?», E. Schwertheim - E. Winter (Hrsg.), Religion und Region. Götter und Kulte aus dem östlichen Mittelmeerraum, AMS 45, Bonn 2003, 77-91.

\section{Di Stefano 1994}

G. Di Stefano, s.v. Palike, in G. Di Stefano M.I. Gulletta (a cura di), Bibliografia Topografica della Colonizzazione Greca in Italia e nelle Isole Tirreniche 13, Pisa-Roma 1994, 280-282.

\section{Dreher 2010}

M. Dreher, "Gerichtsverfahren vor den Göttern? - ,judicial prayers" und die Kategorisierung der defixionum tabellae», in G. Thür (Hrgs.), Symposion 2009, Akten der Gesellschaft für Griechische und Hellenistische Rechtsgeschichte Bd. 21, Wien, 303-337.

DreHER 2012

M. Dreher, "„Prayers for Justice“ and the Categorization of Curse Tablets», in M. Piranomonte - F. Marco Simón (eds.), Contextos Mágicos - Contesti Magici (Atti del Convegno Internazionale Roma 4 - 6 novembre 2009), Roma 2012, 29-32.

EGER 1939

O. Eger, «Eid und Fluch in den maionischen und phrygischen Sühne-Inschriften», in M. Kaser (Hrsg.), Festschrift Paul Koschaker, Böhlau-Weimar 1939, 281-293.

Engels - VAn Nuffelen 2014

D. Engels - P. Van Nuffelen (eds.), Competition and Religion in Antiquity, Collection Latomus 343, Brussels 2014.

FeldTKeller 1994

A. Feldtkeller, Im Reich der syrischen Göttin. Eine religiös plurale Kultur als Umwelt des frühen Christentums, Studien zum Verstehen fremder Religionen 8, Gutersloh 1994.

Fehrle 2010

E. Fehrle, Die kultische Keuschheit im Altertum, Gießen 1910.

FERRI 2010

G. Ferri, Tutela Urbis - Il significato e la concezione della divinità tutelare cittadina nella religione romana, Potsdamer Altertumswissenschaftliche Beiträge 32, Stuttgart 2010.

Franco 1999

A. Franco, «La "città" del Mendolito: Toıvakoín?», Sicilia Archeologica 32 (1999), 99-101.

FRANCO 2008

A. Franco, Periferia e frontiera nella Sicilia antica. Eventi, identità a confronto e dinamiche antropiche nell'area centro-settentrionale fino al IV sec. a.C., Supplementi a Kokalos 19, Roma 2008.

Funke-HaAke 2013

P. Funke - M. Haake (eds.), Greek Federal States and Their Sanctuaries. Identity and Integration, Stuttgart 2013.

FurLANI 1936

G. Furlani, La religione degli Hittiti, Bologna 1936.

GaGLiano 2012

E. Gagliano, «Il dio Adrano: riletture e riflessioni», in T. Alfieri Tonini (a cura di), Mythoi siciliani in Diodoro. Atti del seminario di studi, Aristonothos. Scritti per il Mediterraneo antico, 7, 2012, 301-319.

\section{GaLlazZI 1985}

C. Gallazzi, «Supplica ad Atena su un ostrakon da Esna", Zeitschrift für Papyrologie und Epigraphik 61 (1985), 101-109.

Galvagno 1991

E. Galvagno, «Ducezio "eroe”: storia e retorica in Diodoro", in E. Galvagno - C. Molè Ventura (a cura di), Mito storia tradizione. Diodoro siculo e la storiografia classica, in Atti del Convegno internazionale (Catania - Agira 7-8 dicembre 1984), Catania 1991, 99-124.

GARLAND 1992

R. Garland, Introducing New Gods. The Politics of Athenian Religion, London 1992.

Gernet 1948-1949

L. Gernet, «Droit et prédroit en Grèvce ancienne», L'Année sociologique (1940/1948), Troisième série, T. 3, (1948-1949), 21-119. 
Giammellaro 2008

P. Giammellaro, "Religione e religioni della Sicilia antica nell'opera di Emmanuele Ciaceri», Studi e Materiali di Storia delle Religioni 74 (2008), 49-76.

GLADIGOW 2005

B. Gladigow, Religionswissenschaft als Kulturwissenschaft. Herausgegeben von C. Auffahrt - J. Rüpke, Stuttgart 2005.

Gnoli-Thornton 1997

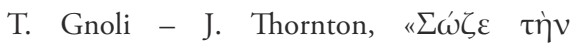
$\kappa \alpha \tau$ токí $\alpha v$. Società e religione nella Frigia romana. Note introduttive», in R. Gusmani-M. Salvini - P. Vannicelli (a cura di), Frigi e Frigio. Atti del 1 Simposio Internazionale (Roma 16-17 Ottobre 1995), Roma 1997, 153-200.

Gordon 2004a

R. Gordon, «Raising a sceptre: confessionnarratives from Lydia and Phrygia», Journal of Roman Archaeology 17 (2004), 177-196.

Gordon 2004b

R. Gordon, «Social Control in the Lydian and Phrygian 'Confession' Texts», in L. Hernández Guerra - J. Alvar Ezquerra (eds.), Actas del XXVII Congreso Internacional Girea-Arys IX. Jerarquias religiosas y control social en el mundo antiguo (Valladolid, 7-9 de Noviembre 2002), Valladolid 2004, 193-203.

GraF 2001

F. Graf, «Fluch und Segen. Ein Grabepigramm und seine Welt», in S. Buzzi - D. Käch - E. Kistler - E. Mango - M. Palaczy - O. Stefani (Hrsg.), Zona archeologica. Festschrift für Hans Peter Isler zum 60. Geburtstag, Bonn 2001, 183-191.

Graf 2004a

F. Graf, «Trick or Treat? On Collective Epiphanies in Antiquity», Illinois Classical Studies 29 (2004), 111-130.

GraF 2004b

F. Graf, "Confession, Secrecy, and Ancient Societies», in Luchesi B. - K. Von Stuckrad (Hrsg.), Religion im kulturellen Diskurs. Festschrift für Hans G. Kippenberg zu seinem 65. Geburstag, Berlin-New York 2004, 259-271.
Graf 2009

F. Graf, «Untimely Death, Witchcraft, and Divine Vengeance. A Reasoned Epigraphical Catalog», Zeitschrift für Papyrologie und Epigraphik 162 (2009), 139-150.

Greco-Lombardo 2012

E. Greco - M. Lombardo, «La colonizzazione greca: modelli interpretativi nel dibattito attuale», in M. Lombardo (a cura di.), Alle origini della Magna Grecia. Mobilità, migrazioni, fondazioni, Atti del $5^{\circ}$ convegno internazionale di studi sulla Magna Grecia (Taranto, 1-4 ottobre 2010), Taranto 2012, 37-60.

\section{GuarducCi 1978}

M. Guarducci, Epigrafia greca IV. Epigrafi sacre, pagane e cristiane, Roma 1978.

GuÉRAud 1932

O. Guéraud, ENTEUXEIS. Requetes et plaintes au roi d'Égypte au IIIe siècle avant J.-C., Le Caire 1932.

HAUKEN 1998

T. Hauken, Petition and Response. An Epigraphic Study of Petitions to Roman Emperors 181-249, Bergen 1998.

HäUSSLER 2013

R. Häussler, Becoming Roman? Diverging Identities and Experiences in Ancient Northwest Italy, University College London Institute of Archaeology Publications 57, Walnut Creek 2013.

Herrmann 1990

P. Herrmann, Hilferufe aus römischen Provinzen. Ein Aspekt der Krise des römischen Reiches im 3. Jhdt. n.Chr., Hamburg 1990.

Herrmann - Malay 2007

P. Herrmann - H. Malay, New Documents from Lydia, Ergänzungsbände zu den Tituli Asiae Minoris 24, Wien 2007.

HÜBNER 2003

S. Hübner, «Spiegel und soziale Gestaltungskraft alltäglicher Lebenswelt: Der Kult des Men in Lydien und Phrygien", in E. Schwertheim - E. Winter (Hrsg.), Religion und Region. Götter und Kulte aus dem östlichen Mittelmeerraum, AMS 45, Bonn 2003, 179-200. 
ISLER 2012

H.P. Isler, Monte Iato, un insediamento arcaico con popolazione etnica mista, in Convivenze etniche, scontri e contatti di culture in Sicilia e Magna Grecia, Aristonothos. Scritti per il Mediterraneo antico, 7, 2012, 113-132.

JES 2007

K. Jes, «Eine Stadt von edler Abkunft und hohem Alter. Kulturelles Gedächtnis in Aizanoi im 2. Jh. n. Chr.», in O. Cordovana - M. Galli (a cura di), Arte e memoria culturale nellétà della seconda sofistica, Catania 2007, 153-168.

La Rosa 1989

V. La Rosa, «Le popolazioni della Sicilia: Sicani, Siculi, Elimi», in G. Pugliese Carratelli (a cura di), Italia omnium terrarum parens, Milano $1989,3-112$.

La Rosa 1996

V. La Rosa, "L'incontro dei coloni greci con le genti anelleniche della Sicilia», in G. Pugliese Carratelli (a cura di), I Greci in Occidente. Catalogo della mostra, Milano 1996, 523-532.

KAISER 2006

T. Kaiser, «In search of Oriental cults. Methodological problems concerning 'the particular' and 'the general' in Near Eastern religion in Hellenistic and Roman periods", Historia 55 (2006), 26-47.

KAISER 2008

T. Kaiser (ed.), The Variety of Local Religious Life in the Near East in the Hellenistic and Roman Periods, Religions in the Graeco-Roman World 164, Leiden-Boston 2008.

Kerschbaum 2014

S. Kerschbaum, «Apollon Lairbenos und seine lokalen Epiklesen auf den Bronzemünzen des phrygischen Hierapolis», Jahrbuch für Numismatik und Geldgeschichte 64 (2014), 15-42.

KieRnAN 2004

P. Kiernan, "Britische Fluchtafeln und „Gebete um Gerechtigkeit" als öffentliche Magie und Votivritual», in K. Brodersen - A. Kropp (Hrsg.), Fluchtafeln. Neue Funde und neue Deutungen zum antiken Schadenzauber, Frankfurt a.M. 2004, 99-114.
KLAUCK 1996

H.-J. Klauck, «Die kleinasiatischen Beichtinschriften und das Neue Testament», in H. Cancik - P. Schäfer - H. Lichtenberger (Hrsg.), Geschichte - Tradition - Reflexion. Festschrift für M. Hengel, Tübingen 1996, 63-87 (=H.J. Klauck, Religion und Gesellschaft im frühen Christentum, Tübingen 2003, 57-115)

Kotsifou 2012

C. Kotsifou, «Emotions and Papyri. Insights into the Theatre of Human Experience in Antiquity», in A. Chaniotis (ed.), Unveiling Emotions. Sources and Methods for the Study of Emotions in the Greek World, HABES 52, Stuttgart 2012, 39-90

KROPP 2008

A. Kropp, Magische Sprachverwendung in vulgärlateinischen Fluchtafeln (defixiones), Tübingen 2008.

LAMAGNA 2009a

G. Lamagna, "Adrano, piazza Eurelios: saggi nell'area della necropoli est", in Lamagna 2009b, 129-144

LAMAGNA 2009b

G. Lamagna, (ed.), Tra Etna e Simeto. La ricerca archeologica ad Adrano e nel suo territorio, Catania 2009

LAMAGNA 2011

G. Lamagna, «L'insediamento greco di Adrano tra Timoleonte e Ierone II: i dati delle ultime ricerche», in M. Congiu - C. Micciché - S. Modeo (a cura di), Timoleonte e la Sicilia della seconda metà del IV sec. a. C., Atti del VII Convegno di studi, Caltanissetta 2011, 57-73.

LURAGHI 1994

N. Luraghi, Tirannidi archaiche in Sicilia e in Magna Grecia, Firenze 1994.

MA 2008

J. Ma, «Paradigms and Paradoxes in the Hellenistic World», Studi ellenistici 20 (2008), 371385.

Malay - Petzl 2017

H. Malay - G. Petzl, New religious texts from Lydia, Wien 2017. 
Manganaro 1961

G. Manganaro, «Iscrizioni di Adrano in alfabeto siculo", Archeologia Classica 13 (1961), 106-112.

\section{MaLKIN 1998}

I. Malkin, "The Middle Ground: Pholoktetes in Italy», Kernos 11 (1998), 131-141.

\section{MaLKIN 2002}

I. Malkin, «A colonial middle ground. Greek, Etruscan and local Archaeology in the Bay of Neaples», in C. Lyons - J. Papadopoulos (eds.), The Archaeology of Colonialism, Los Angeles 2002, 151-181.

MALKIN 2004

I. Malkin, «Postcolonial Concepts and ancient Greek colonization», in B. Fuchs - D.J. Parker (eds.), Colonialism and the Past, Modern Language Quaterly 65 (2004), 341-364.

MALKIN 2011

I. Malkin, A Small Greek World. Networks in the Ancient Mediterranean, Oxford 2011.

Maniscalco-McConnell 1997-1998

L. Maniscalco - B. McConnell, «Ricerche e scavi intorno a Paliké», Kokalos 43-44 (19971998), 173-188.

Maniscalco - McConnell 2003

L. Maniscalco - B. McConnell, «The Sanctuary of the Divine Palikoi (Rocchicella di Mineo, Sicily): Fieldwork from 1995 to 2001", American Journal of Archaeology 107 (2003), 145-180.

Maniscalco 2008

L. Maniscalco, Il santuario dei Palici: un centro di culto nella Valle del Margi, Palermo 2008.

Manni 2004

E. Manni, Geografia fisica e politica della Sicilia antica, Roma $2004^{2}$.

Marchesini 1999

S. Marchesini, La situazione alfabetica: l'Italia meridionale e la Sicilia, in Confini e frontiera nella Grecità d'Occidente. Atti del trentasettesimo convegno di studi sulla Magna Grecia (Taranto 3-6 ottobre 1997), Napoli 1999, 173-212.
Marino-Stavru 2015

S. Marino - A. Stravru (eds.), Ekphrasis, Estetica. Studi e ricerche 1, Roma 2015.

MicCICHÈ 2011

C. Miccichè Mesogheia. Archeologia e storia della Sicilia centro-meridionale dal VII al IV sec. a.C., Caltanisetta $2011^{2}$.

Millar 1987

F. Millar, «Empire, Community and Culture in the Roman Near East: Greeks, Syrians, Jews and Arabs», The Journal of Jewish Studies 38 (1987), 143-164.

I. Mörth, s.v. Kommunikation, in Handbuch religionswissenschaftlicher Grundbegriffe III, Stuttgart 1993, 392-414.

MoraWIECKi 1995

L. Morawiecki, «Adranos. Una divinità dai molteplici volti», Kokalos 41 (1995), 29-50.

ÖHLINGER 2015

B. Öhlinger, Ritual und Religion im archaischen Sizilien. Formations- und Transformationsprozesse binnenländischen Kultorte im Kontext kultureller Kontakte, Italiká 4, Wiesbaden 2015.

PACE 1935

B. Pace, Arte e civiltà della Sicilia antica I. I fattori etnici e sociali, Milano 1935.

PARKer 1983

R. Parker, Miasma. Pollution and purification in early Greek religion, Oxford 1983.

PARker 2004

R. Parker, «What Are Sacred Laws?», in E.M. Harris - L. Rubinstein (eds). The Law and the Courts in Ancient Greece, London 2004, 57-70.

Pedridou 2015

G. Pedridou, Divine Epiphany in Greek Literature and Culture, Oxford 2015.

Petrovic - Petrovic 2016

A. Petrovic - I. Petrovic, Inner purity and pollution in Greek religion. 1. Early Greek religion, Oxford 2016.

Pettazzoni 1936

R. Pettazzoni, La confessione dei peccati II, 3, Bologna 1936. 
Pettazzoni 1937

R. Pettazzoni, "Confession of sins and classics», Harward Theological Review 30 (1937), 1-14 (= Essays on the History of Religions, Leiden 1967, 55-67).

Pettazzoni 1955

R. Pettazzoni, L'onniscienza di dio, Torino 1955.

Petzl 1994

G. Petzl, «Die Beichtinschriften Westkleinasiens», Epigraphica Anatolica 22 (1994).

Petzl 1995

G. Petzl, «Ländliche Religiosität in Lydien», in

E. Schwertheim (Hrsg.), Forschungen in Lydien. AMS 17, Bonn, 37-48.

\section{Petzl 1998}

G. Petzl, Die Beichtinschriften im römischen Kleinasien und der Fromme und Gerechte Gott, Wiesbaden 1998.

Petzl 2002

G. Petzl, «Zum religiösen Leben im westlichen Kleinasien: Einflüsse und Wechselwirkungen", in H. Blum H. - B. Faist - P. Pfälzner - A.M- Wittke (Hrsg.), Brückenland Anatolien? Ursachen, Extensität und Modi des Kulturaustausches zwischen Anatolien und seinen Nachbarn, Tübingen, 381-391.

Petzl 2007

G. Petzl, Tituli Lydiae Linguis Graeca et Latina Conscripti: Fasciculus III, Philadelpheia et ager Philadelphenus (Tituli Asiae Minoris), Wien 2007.

Platt 2015

V. Platt, Facing the gods: epiphany and representation in Graeco-Roman art, literature and religion, Cambridge 2011.

Pleket 1981

H.W. Pleket, «Religious History as the History of Mentality: the ,Believer' as Servant of the Deity in the Greek World», in H.S. Versnel (ed.), Faith, Hope and Worship: Aspects of Religious Mentality in the Ancient World, Studies in Greek and Roman Religion 2, Leiden 1981, 152-192.

Rizzo 2012

R. Rizzo, Culti e miti della Sicilia antica e protocristiana, Caltanisetta 2012.
Rostad 2002

A. Rostad, "Confession or Reconciliation? The Narrative Structure of the Lydian and Phrygian ,Confession Inscriptions'", Symbolae Osloenses 77 (2002), 145-164.

RÜPKE 2010

J. Rüpke, «Hellenistic and Roman Empires and Euro-Mediterranean Religion", Journal of Religion in Europe 3 (2010), 197-214.

RÜPKE 2011

J. Rüpke, «Reichsreligion? Überlegungen zur Religionsgeschichte des antiken Mittelmeerraums in der römischen Zeit", Historische Zeitschrift 292 (2011), 297-322.

\section{SALvo 2012}

I. Salvo, «Sweet Revenge. Emotional Factors in 'Prayers for Justice'», in A. Chaniotis (ed.), Unveiling Emotions. Sources and Methods for the Study of the Emotions in the Greek World, HABES 52, Stuttgart 2012, 235-266.

SAMmartano 1998

R. Sammartano, Origines gentium Siciliae: $E l-$ lanico, Antioco, Tucidide, Supplementi a Kokalos 14, Roma 1998.

Scheid-de Polignac 2010

J. Scheid - F. de Polignac, "Qu'est-ce qu'un "paysage religieux»? Représentations cultuelles de l'espace dans les sociétés anciennes», Revue de l'histoire des religions 4 (2010), 427-434.

SCHÖRNER 2005

G. Schörner, "Das Zentrum - Peripherie Modell in der Romanisierungsforschung», in G. Schörner (Hrsg.), Romanisierung - Romanisation: Theoretische Modelle und praktische Fallbeispiele. Beiträge eines Graduiertenkolloquiums an der Friedrich-Schiller Universität Jena 2003/2004, BAR International Series 1427 , Oxford 2005, 95-99.

Sima 1999

A. Sima, «Kleinasiatische Parallelen zu den altsüdarabischen Buß- und Sühneninschriften», Altorientalische Forschungen 26 (1999), 140-153.

Simonetti Agostinetti 2012

A. Simonetti Agostinetti, «Ducezio e il mito 
della polis", in Convivenze etniche, scontri e contatti di culture in Sicilia e Magna Grecia, Aristonothos. Scritti per il Mediterraneo antico, 7 (2012), 321-333.

\section{SoKOLOWSKI 1962}

F. Sokolowski, Lois sacrées des cités grecques: supplement, Paris 1962.

\section{SoKolOwsKI 1969}

F. Sokolowski, Lois sacrées des cités grecques, $\mathrm{Pa}-$ ris 1969.

\section{Sommer 2012}

M. Sommer, «Heart of Darkness? Post-colonial theory and the transformation of the Mediterranean", Ancient West \& East 11 (2012), 235245.

\section{Sordi 1980}

M. Sordi, «Il IV e il III secolo da Dionigi I a Timoleonte (336 a.C.)», in E. Gabba - G. Vallet (a cura di), La Sicilia antica, Napoli 1980, 209-288.

\section{SPATAFOra 2010}

F. Spatafora, «Per un' "archeologia degli incontri": Sicani ed Elimi nella Sicilia greca", in H. Tréziny (éd.), Grecs et Indigenes de la Catalogne à la mer Noire, Actes des rencontres du programme européen Ramses 2 (2006-2008), Paris 2010, 25-40.

\section{Spatafora 2012}

F. Spatafora, «Interrelazioni e commistioni nella Sicilia nord-occidentale di età arcaica: i contesti funerari come indicatori archeologici», in Convivenze etniche, scontri e contatti di culture in Sicilia e Magna Grecia, Aristonothos. Scritti per il Mediterraneo antico, 7 (2012), 59-90.

Spatafora 2014

F. Spatafora, «Formazione e caratteri identitari delle comunità indigene nella Sicilia occidentale», in G. Greco - B. Ferrara (a cura di), Segni di appartenenza e identità di comunità nel mondo indigeno. Atti del Seminario di Studi (Napoli 6-7 luglio 2012), Napoli 2014, 369-383.

\section{StaVrianopoulou 2012}

Stavrianopoulou E. 2012, «tou dikaiou tychein oder die Macht der Bitte», in C. Kuhn (Hrsg.), Politische Kommunikation und Öffentliche Mei- nung in der Antiken Welt, Stuttgart 2012, 123 149.

StAVRianopoulou 2013

E. Stavrianopoulou, «Introduction», in E. Stavrianopoulou (ed.), Shifting Social Imaginaries in the Hellenistic Period. Narrations, Practices, and Images, Supplementum Mnemosyne 363, Leiden-Boston 2013, 1-21.

Stramaglia 1999

A. Stramaglia, Res inauditae, incredulae. Storie di fantasmi nel mondo greco-latino, Bari 1999.

Strubbe 1997

J. Strubbe, APAI EПITҮMBIOI. Imprecations against Desecrators of the Grave in the Greek Epitaphs of Asia Minor. A Catalogue, IK 52, Bonn 1997.

TADDEI 2009

A. Taddei, «Il prediritto come funzione psicologica", in Studi in onore di Remo Martini III, Milano 2009, 499-522.

Totaro 2011

P. Totaro, «La fondazione di Etna e le reliquiae delle Etnee», in A. Beltrametti (a cura di), $\mathrm{La}$ storia sulla scena. Quello che gli storici antichi non hanno raccontato, Roma 2011, 149-168.

Тотті 1985

M. Totti, Ausgewählte Texte der Isis- und Sarapis-Religion, Hildesheim 1985.

ToMLin 1988

R.S. Tomlin, «Roman Inscribed Tablets of Tin and Lead from the Sacred Spring at Bath", in B. Cunliffe (ed.), The Temple of Sulis Minerva at Bath. Volume 2. The Finds from the Sacred Spring, Oxford 1988, 59-277.

Tomlin 2010

R.S. Tomlin, "Cursing a thief in Iberia and Britain", in R. Gordon (ed.), Magical practice in the Latin West: papers from the international conference held at the University of Zaragoza (30 Sept.1 Oct. 2005), Religions in the Graeco-Roman World 168, Leiden-Boston 2010, 245-273.

Traina 2006

Traina G. 2006, «Romanizzazione, "métissages", ibridità», Mélanges de l'Ecole française de Rome. Antiquité 118 (2006), 151-158. 
VASSALLO 1999

S. Vassallo, «Metalli», in S. Vassallo (a cura di), Colle Madore. Un caso di ellenizzazione in terra sicana, Palermo 1999, 90-116.

Versnel 1987a

H.S. Versnel, «Les imprécations et le droit», Revue historique de droit français et étranger 65 (1987), 3-32.

\section{VERSNEL 1987b}

H.S. Versnel, «What did Ancient Man when he saw a God? Some Reflections on Greco-Roman Epiphany», in D. Van Der Plas (ed.), Effigies Dei. Essays on the History of Religions, Leiden, 42-55.

VERSNEL 1991

H.S. Versnel, «Beyond the Cursing: The Appeal to Justice in Judicial Prayers», in C. Faraone D. Obbink (eds.), Magika Hiera. Ancient Greek Magic and Religion, New York - Oxford 1991, 60-106.

VERSNEL 1994

H.S. Versnel, «ПЕПРНMENOE. The Cnidian Curse Tablets and Ordeal by Fire», in R. Hägg (ed.), Ancient Greek Cult Practice from the Epigraphical Evidence. Proceedings of the Second International Seminar on Ancient Greek Cult, organised by the Swedish Institute at Athens (22-24 November 1991), Stockholm 1994, 145-154.
VERSNEL 2002

H.S. Versnel, «Writing Mortals and Reading Gods. Appeal to the Gods as a Strategy in Social Control», in D. Cohen (ed.), Demokratie, Recht und soziale Kontrolle im klassischen Athen, Schriften des Historischen Kollegs. Kolloquien 49, München 2002, 37-76.

VERSNEL 2009

H.S. Versnel, Fluch und Gebet. Magische Manipulation versus religiöses Flehen?, HansLietzmann-Vorlesungen 10, Berlin 2009.

VERSNEL 2012

H.S. Versnel, "Response to a Critique», in M. Piranomonte - F. Marco Simón (eds.), Contextos Mágicos - Contesti Magici. Atti del Convegno Internazionale (Roma 4 - 6 novembre 2009), Roma 33-45.

White 2011

R. White, The Middle Ground. Indians, Empires and Republics in the Great Lakes Region, 16501815, Cambridge 2010.

WILLI 2008

A. Willi, Sikelismos: Sprache, Literatur und Gesellschaft im griechischen Sizilien (8. - 5. Jh. v. Chr.), Basel 2008.

WOOWARD - LEACH 1993

A. Wooward - P. Leach (eds.), The Uley Shrines: Excavation of a Ritual Complex on West Hill, Uley, Gloucestershire: 1977-9, Oxford 1993. 